Universität Bielefeld Faculty of Business Administration and Economics

Working Papers in Economics and Management

No. 04-2013

March 2013

\title{
Best response dynamics with level- $n$ expectations in two-stage games
}

Herbert Dawid

Dennis Heitmann 


\title{
Best response dynamics with level- $n$ expectations in two-stage games
}

\author{
Herbert Dawid $^{*} \quad$ Dennis Heitmann ${ }^{\dagger}$
}

December 2012

\begin{abstract}
This paper analyzes behavior in repeatedly played two-stage games, where players choose actions in both stages according to best replies using 'level- $n$ expectations' about the opponent's actions in both stages. Level- $n$ expectations are recursively defined in a way that a player holding level $n$ expectations correctly predicts the action of an opponent holding level $n-1$ expectations. A general conceptual framework to study such dynamics for two-stage games is developed and it is shown that, contrary to results for single-stage games, the fixed points of the dynamics depend on the level of the expectations. In particular, for level-zero expectation, fixed points correspond to a Nash equilibrium of a simultaneous move version of the game, whereas (under certain conditions) fixed points converge towards the subgame perfect equilibrium of the two-stage game if the level of expectations goes to infinity. The approach is illustrated using a two-stage duopoly game, where firms in the first stage invest in activities reducing their marginal costs and in the second stage engage in Cournot competition. An increase in the level of expectations leads in the long run to higher cost reducing activities and higher output of the firms, however to lower profits. Level-two expectations are sufficient to move the fixed-point of the dynamics to a close neighbourhood of the subgame-perfect equilibrium.
\end{abstract}

Keywords: expectation formation; multi-stage games; best response dynamics JEL classification: C72; C73; D83; L13

*Department of Economics and Business Administration and Institute of Mathematical Economics, Bielefeld University, P.O. Box 100131, 33501 Bielefeld, Germany, e-mail: hdawid@wiwi.unibielefeld.de

${ }^{\dagger}$ Department of Economics and Business Administration, Bielefeld University, P.O. Box 100131, 33501 Bielefeld, Germany, e-mail: dheitmann@wiwi.uni-bielefeld.de 


\section{Introduction}

A large part of the theoretical and empirical analysis in many areas of economics relies on the assumption that the behavior of the involved decision makers is determined by some sort of equilibrium. In contexts, like Industrial Organization, where multi-stage games are frequently used to describe strategic interaction with sequential decisions, a standard equilibrium concept considered in the literature is that of subgame perfect equilibrium as a refinement of Nash equilibrium. The notion of (Nash) equilibrium rests on two basic assumptions. First, the assumption that each player is able to determine his payoff-maximizing strategy given the strategies of all other players and, second, that in equilibrium the expectations of each player concerning the strategies of all players coincide with the strategies actually used by these players. Both assumptions have been extensively discussed in the literature and considerable amount of research has analyzed the question under which circumstances players, who ex-ante cannot determine their best responses and/or do not have correct expectations about the strategies followed by the other players, can over time coordinate in a way such that eventually a Nash equilibrium is played. These studies assume that the considered game is played repeatedly and that players adjust their strategies over time according to some given process.

The earliest stream of literature dealing with the dynamic stability of Nash equilibria is work on best response dynamics, where it is assumed that each player is able to determine his best response correspondence, but ex-ante expectations about the other players strategy are in general not correct and are updated over time (see. e.g. Brown (1951)). A rich literature has studied the dynamic properties of best response dynamics under naive and adaptive expectations in the framework of oligopolistic market interaction (see the survey in Kopel (2009)) identifying conditions on the market environment and the expectation formation process under which Cournot, respectively Bertrand, equilibria are (locally asymptotically) stable fixed points of the corresponding best response dynamics. This entire literature on best response dynamics in oligopolies focuses, however, on one-stage games, which is quite restrictive since many important issues in oligopolistic competition, like capacity choice, location choice or cost reducing activities of firms, are typically analyzed using multi-stage games.

The agenda of this paper is to relax this restriction to one-stage games and to extend the analysis of best response dynamics to a scenario where in each period firms interact in two sequential stages. Furthermore, we relax the assumptions of naive expectations of individuals, which is typically made in this literature, to a class of expectation formation functions denoted as 'level- $n$ expectations, which include 
naive expectations as a special case.

In particular, we consider a standard two-stage model of duopolistic competition with differentiated products introduced in Qiu (1997), where in the first stage firms make cost-reducing investments and, in the second stage, they sell the product in the market. We consider a scenario where the demand function and the cost structure of both firms is common knowledge, but firms face strategic uncertainty in a sense that they do not know their opponents actions in the current stage when making their decision. Hence, they build expectations about these actions and then choose their best response given these expectations. First stage actions are observable between the stages, which means that expectations about the opponents second stage action might be influenced by the first stage action of this player. Given our assumption that the payoff functions of both players are common knowledge, both players are in a position to determine not only the own best response function but also that of the opponent in both stages.

Consider now a firm which expects that its competitor uses naive expectations about its own actions. Anticipating that this competitor will play its best repsonse based on these naive expectations the firm is able to correctly predict the action of its competitor for any realization of the previous period actions (since the previous period actions are common knowledge). Denoting naive expectations as level-0 expectations, we will say that a firm following this kind of rationale has level-1 expectations. Such a firm would then choose its actions as a best response to its level-1 expectations of the opponents action. Any firm who would correctly predict the actions a firm with level-1 expectations is said to have level-2 expectations and extending this reasoning we define level- $n$ expectations for an arbitrary integer $n$.

The consideration of best response dynamics under expectations of this kind in a two-stage setting raises several questions. First, and foremost, the issue of equilibrium selection arises. Oligopoly games with multiple stages typically have multiple Nash equilibria, among which there is a unique subgame perfect equilibrium. It is a standard result that the fixed points of iterated best reply dynamics (under naive expectations) are Nash equilibria of the underlying game, but in the presence of multiple equilibria it is quite unclear which of these equilibria are (stable) fixed points of the dynamics. The question arises whether and how the set of fixed points of the best reply dynamics under level- $n$ expectations depends on the value of $n$. In particular, we are interested in understanding under which circumstances firms may in the long run coordinate on a subgame perfect equilibrium of the underlying twostage game. Second, extending the expectation formation process described above from a one-stage to a two-stage game setting is not straightforward. We consider a 
two-stage game, where first stage actions are common knowledge in the second stage. This implies not only that the expectation of a firm with respect to its opponents' action in stage two should be updated after the revelation of the opponent's action in stage one, but also that the firm should have expectations about the effect of its own first stage action on its opponent's action in the second stage. The opponents action in stage two is affected through two channels. First, the best response of the opponent for a given expectation about the own stage 2 action might be influenced by the own stage 1 action. Given our assumption that the firm knows the payoff function and hence the second stage best response function of the opponent this effect of the own stage 1 action is easy to predict. Second, the revelation of the own first stage action might influence the opponents expectations about the own action in stage two, which in turn affect the best response action of the opponent. This channel of influence on the opponents stage-two choice depends on the way the opponents expectation formation function. Hence, the best response function of a firm on stage two depends on the way expectations are formed on stage two, in particular it depends on the level of expectation formation on that stage. Such a dependency of the best response function of a firm on the way expectations are formed does not arise in the framework of one stage games and this induces that the properties of the considered dynamics in two-stage games are stronger affected by the level of expectation formation.

The paper puts forward three main findings. First, it is shown for a general class of two-stage games, which includes the duopoly model with cost reducing investments as well as many other standard models in industrial organization, that, if the expectation formation follows the standard assumption of level-zero (i.e. naive) expectations, then the fixed point of the best response dynamics corresponds to a Nash equilibrium of a version of the game, where first stage actions are not revealed before the second stage. Such equilibria are in general not subgame perfect. Put differently, strategic effects of first stage actions on second stage outcomes have no role under level zero exepctations. Second, it is shown again for the general class of games that such strategic effects do play a role for the determination of the fixed point of the dynamics if firm have expectations of higher level. As the level of expectations tends to infinity the fixed points of the dynamics converge to subgame perfect equilibrium outcomes. In the framework of the duopoly with cost reducing activities it is demonstrated that already for level-2 expectations the unique fixed point of the dynamics is very close to the unique subgame perfect equilibrium. Third, if the level of expectation formation goes up in the duopoly with cost reducing activities this induces an increase in the long run consumer surplus 
however a decrease in the long run profits of the firms.

These results are helpful for disentangling two implications of the revelation of first-stage actions before the second stage. The first implication of the revelation, which is that a firm can observe the opponent's first stage action before choosing the own second stage action, turns out to be irrelevant for the fixed point of the best reply dynamics if expectations on stage 2 are naive. Although firms for any level of expectations fully take the observed opponent's first stage action into account when determining their best reply, for expectation level smaller than two the fixed point coincides with the one of the corresponding best reply dynamics, where the first-stage actions are not revealed in the second stage Crucial for the location of the fixed point of the dynamics is the second implication of the revelation of first stage actions, namely that firms expect that their own first stage action influences the opponents second stage choice via the opponents second stage expectation formation. This effect becomes stronger the higher the level of the expectations and for large expectation levels approaches the full fledged inter-stage strategic effect present in the subgame perfect equilibrium.

The organization of the paper is as follows. In section 2 we review the related literature. In section 3 we introduce the concept of level- $n$ expectation in a general setting and derive in this framework characterizations of the steady states of the best response dynamics under level- $n$ expectations. Section 4 studies the implications of an increase of the expectations level in a dynamic duopoly model, where firms can invest in cost-reducing activities before competing on the market. Finally, section 5 concludes the paper. All proofs are given in the Appendix.

\section{Related Literature}

The paper is related to several streams of literature, which we briefly review here.

First, the question under which circumstances boundedly rational firms coordinate on subgame perfect equilibria of extensive form games, has been discussed for different types of evolutionary dynamics governing the strategy updating of players. Noeldecke and Samuelson (1993) consider a model, where for every player of an extensive form game a population of individuals exists where every possible combination of individuals meets and plays. Individuals have beliefs about the frequency of actions at all information sets and update them based on observable action profiles. They determine their strategy as best responses to their beliefs in all information sets, where with a small mutation probability the strategy is chosen randomly from a given distribution. For extensive form games with perfect information, where 
every player moves at most once, the subgame perfect equilibrium outcome has positive weight in the limiting distribution of the resulting Markov chain for mutation probabilities going to zero. However, in general also Nash equilibrium outcomes which are not subgame perfect can have positive weight in the limiting distribution, which means that evolution generally speaking does not 'select' the subgame perfect equilibrium. Intuitively, this result is due to a potential drift of individuals' beliefs about action profiles in information sets that are not reached in the subgame perfect equilibrium. As has been shown in subsequent papers by Hart (2002) and Kuzmics (2004), a unique selection of subgame perfect equilibrium in a certain sense can be achieved if it is assumed that the population size tends to infinity as mutation probabilities become small. Although these papers also deal with best response dynamics of certain type, the considered setup is quite different from ours. Differently from our setup, a population of individuals is considered for each player, where individuals base their beliefs completely on observed action profiles rather than taking into account best response correspondences of the other players, as they do in our setup. This property together with the presence of stochastic mutations are the key factors allowing for the drift of beliefs in off-equilibrium information sets. Under the level- $n$ expectation dynamics considered here, players rely on best response considerations when building their beliefs and therefore also for information sets, which have not been reached in previous periods beliefs about opponents actions cannot drift. The qualitative insights that subgame perfect equilibria of perfect information games are stable with respect to evolutionary dynamics, but in general not the unique stable outcome, has also been derived in the framework of different classes of deterministic selection dynamics in Cressman and Schlag (1998) and Demichelis and Ritzberger (2003).

The second related stream of literature deals with best response dynamics in oligopolies. Such dynamics have been studied extensively under the assumption of naive expectations (e.g. Theocharis (1960), Puu (1998), Tramontana et al. (2009)) and adaptive expectations (e.g. Okuguchi (1970), Bischi and Kopel (2001)), for different assumptions about demand and production structure. Fixed points in these one-stage settings always correspond to Cournot respecitvely Bertrand equilibria and the focus of analysis has been on the stability of these fixed points, on the shape of the basins of attractions in the case of several (locally) stable equilibria and on the occurence of complex dynamics in such settings (see e.g. Bischi et al. (2010)). Our results contribute directly to this literature by characterizing properties of the fixed points of best response dynamics under naive expectations (level-zero expectations in our notation) for two-stage games. More generally, our results show that the 
overall insight from this literature that any Nash equilibrium of the game is a fixed point of best response dynamics under 'reasonable' expectation formation rules (e.g. naive or adaptive expectations) does not extend to multi-stage games.

The third stream of literature, which is relevant for this paper, deals with the incentive for cost reducing activities of firms in oligopolistic markets. This literature is based on the insight that cost advantages relative to competitors improve the position of a firms in oligopolistic competition. At least in cases of quantity competition, common knowledge of marginal cost advantages of a firm makes its competitor act less aggressively, thereby adding positive strategic effects to the positive direct effect of cost reduction on profits. Therefore, the firms (strategic) choice of cost reducing activities is an important factor in oligopolistic competition. Qiu (1997) has analyzed cost reducing activities (CRA) of oligopolistic firms in a twostage game setting, where firms first choose the intensity of cost reducing activities and, after the effects of these activities on costs have become common knowledge, in the second stage compete on the market. Based on the analysis of the (unique) subgame perfect equilibrium of the game it is shown that cost reducing activities are stronger under quantity than under price competition. In several papers using similar two-stage games the effect of cooperation between the firms on the first or on both stages of competition have been considered (e.g. D'Aspremont and Jacquemin (1988)). Whereas much of this literature is based on the consideration of static twostage games, a few contributions have explicitly considered the fact that in most oligopolies firms repeatedly interact on the market and can also repeatedly engage in cost reducing activities. Cellini and Lambertini (2009), Cellini and Lambertini (2011) and Breton et al. (2004) consider dynamic oligopolies, where firms, which at each point in time compete on an oligopolistic market, can over time reduce their marginal costs by investing in R\&D. Since it is assumed here that R\&D investments imply persistent cost reduction, this dynamic strategic interaction is analyzed using differential game models. Contrary to these contributions the focus in this paper is set on cost reducing activities without persistent effects. In order to keep production costs at a certain level firms have to keep also the level of cost reducing activities. Examples for such activities are numerous and include production planning activities, monitoring or quality control activities. Furthermore, contrary to all the contributions discussed above, this paper is based on the assumptions that the cost reducing activities and quantity choices of the firms in the oligopoly ex-ante do not follow any equilibrium of the game, which makes expectation formation an important issue. Best response dynamics under non-rational expectations has been hardly analyzed in the context of $\mathrm{R} \& \mathrm{D}$ choice in oligopolies. A rare exception is 
Bischi and Lamantia (2004) where a dynamic model of R\&D choices is studied under the assumption of naive expectations. However, in their setting competition on the market is represented in reduced form, such that the game repeatedly played by the firms has one-stage structure.

Finally, it should be mentioned that the level- $n$ expectation formation considered in this paper is related to the literature on level- $k$ reasoning initiated in Stahl and Wilson (1995) in the sense that like in this literature we assume that decision makers choose best responses to expectations about the actions of the other players that are recursively built in a way that expectations of order $n$ are determined based on best responses to expectations of order $n-1$. However, the focus of our analysis is quite different to that of the level- $k$ literature. First, we deal with a dynamic model, where decision makers adapt their expectations and actions over time. Whereas the level- $k$ literature is mainly interested in explaining behavior of individuals facing novel situations, we mainly examine the long run behavior emerging if the same type of interaction occurs many times. Second, contrary to the level- $k$ models, there is no hierarchy of beliefs in the best response dynamics with level- $n$ expectations. Rather, all players in the game share the same level of reasoning. Third, to our knowledge the level- $k$ literature has considered only one-shot games, whereas our focus is on two-stage games.

\section{Level- $n$ Expectations and Best Response Dy- namics}

In this section we define level- $n$ expectation and the corresponding best response dynamics for a general class of two-stage games and characterize the steady states of the dynamics for the two extreme cases of $n=0$ and $n \rightarrow \infty$. The games we consider have a (not necessarily unique) interior subgame perfect equilibrium in pure strategies and a unique pure strategy Nash equilibria in each of the subgames on stage two. A large set of standard models in many areas of economics including industrial organization fall into this class of games.

\subsection{A Class of Two-Stage Games}

Consider a two-player two-stage game, where at stage one both players simultaneously choose actions $x_{i} \in X_{i}$ with $X_{i}$ compact subsets of $\mathbb{R}^{m_{i}^{1}}, m_{i}^{1} \geq 1, i=1,2$. Between the two stages, choices made in stage one become common knowledge and at stage two both players simultaneously choose actions $q_{i} \in Q_{i}$ with $Q_{i}$ compact 
subsets of $\mathbb{R}^{m_{i}^{2}}, m_{i}^{2} \geq 1, i=1,2$. At the end of stage two each player receives a payoff $\pi_{i}\left(x_{1}, x_{2}, q_{1}, q_{2}\right) \in \mathbb{R}, i=1,2$. We assume that both payoff functions are everywhere twice continuously differentiable and that $\pi_{i}$ is strictly concave with respect to $q_{i}$ for all $\left(q_{j}, x_{1}, x_{2}\right) \in Q_{j} \times X_{1} \times X_{2}$. Hence, there exist single valued and continuous best response functions on stage two of the form $R_{i}^{q}: Q_{j} \times X_{1} \times X_{2} \mapsto Q_{i}$. Furthermore, we assume that for each pair $\left(x_{1}, x_{2}\right) \in X_{1} \times X_{2}$ the mapping $q \mapsto$ $R_{1}^{q}\left(R_{2}^{q}\left(q_{1}, x_{1}, x_{2}\right), x_{1}, x_{2}\right)$ has a unique fixed point $q \in Q_{1}$, which means that for each subgame defined by $\left(x_{1}, x_{2}\right)$ there exists a unique pure strategy Nash equilibrium. A sufficient condition for this property to hold is that the mapping mentioned above is a contraction and in what follows we will make this assumption. ${ }^{1}$ We define as $q_{i}^{*}: X_{1} \times X_{2} \mapsto Q_{i}$ the choice of player $i$ in the unique Nash equilibrium of the subgame defined by $\left(x_{1}, x_{2}\right) \in X_{1} \times X_{2}$. It follows from the assumptions above that also this mapping is continuous and continuously differentiable for all $\left(x_{1}, x_{2}\right)$ where $q_{i}^{*}\left(x_{1}, x_{2}\right) \in \operatorname{int}\left(Q_{i}\right)$.

Considering the first-stage choices, we define

$$
\pi_{i}^{*}\left(x_{1}, x_{2}\right)=\pi_{i}\left(x_{1}, x_{2}, q_{1}^{*}\left(x_{1}, x_{2}\right), q_{2}^{*}\left(x_{1}, x_{2}\right)\right)
$$

as the payoff of player $i$ induced by a pair of first-stage choices, under the assumption that in all subgames on stage 2 the unique Nash equilibrium is played. We assume that $\pi_{i}^{*}$ is strictly concave with respect to $x_{i}$ for all $\left(x_{1}, x_{2}\right) \in X_{1} \times X_{2}$. A sufficient condition for, often used in applications, is that the concavity of $\pi_{i}$ with respect to $x_{i}$ is sufficiently strong to dominate the effect through $q_{i}^{*}$ and $q_{j}^{*}$. The best response correspondences on stage one are denoted by $R_{i}^{x *}=\arg \max _{x_{i} \in X_{i}} \pi_{i}^{*}\left(x_{1}, x_{2}\right)$ and we assume that the mapping $x_{1} \mapsto R_{1}^{x *}\left(R_{2}^{x *}\left(x_{1}\right)\right)$ has at least one fixed point in $X_{1}$, which means that there exists a subgame perfect equilibrium in pure strategies of the game.

In what follows we will sometimes refer to a version of the two-stage game without revelation of action choices between the stages. In this version of the game the choices made in the first stage remain unknown to the players when they make their decisions in the second stage. Hence, this game is equivalent to a game where the two players choose $x_{i}$ and $q_{i}$ at the same time. A Nash equilibrium $\left(\hat{x}_{1}, \hat{x}_{2}, \hat{q}_{1}, \hat{q}_{2}\right)$ of

\footnotetext{
${ }^{1}$ This assumption could be weakened, but it is made to avoid some technical issues in the proof of the main proposition in the following section. In games where the second stage is characterized by linear best response functions this property is necessary for the existence of a pure strategy Nash equilibrium on stage 2. Hence, in many standard models in different areas of economics a corresponding assumption is made.
} 
this game is characterized by

$$
\hat{x}_{i}=\hat{R}_{i}^{x}\left(\hat{x}_{j}, \hat{q}_{j}\right) \quad \text { and } \quad \hat{q}_{i}=q_{i}^{*}\left(\hat{x}_{1}, \hat{x}_{2}\right), \quad i=1,2,
$$

where

$$
\hat{R}_{i}^{x}\left(x_{j}, q_{j}\right)=\arg \max _{x_{i} \in X_{i}} \pi_{i}\left(x_{i}, x_{j}, R_{i}^{q}\left(q_{j}, x_{i}, x_{j}\right), q_{j}\right)
$$

denotes the optimal choice in the first stage of player $i$, given that the opponent chooses $\left(x_{j}, q_{j}\right)$ and taking into account that the own optimal choice in stage two is given by the best response function on stage two. We refer to such an equilibrium as a Nash equilibrium without revelation of actions between stages. It should be noted that such equilibria are also Nash equilibria of the two-stage game with revelation of actions between stages, but in general they are not subgame perfect equilibria of that game.

\subsection{Best reply dynamics under level- $n$ expectations}

In what follows we consider the dynamic evolution of the behavior of two agents who repeatedly interact by playing the two-stage game described in the previous subsection. They choose their actions at both stages according to their best response with respect to their expectations about the opponent's action. In building their expectations about the opponent's action the players take into account that the opponent will act according to her best response correspondence applied to her expectations. Based on this we introduce the notion of level- $n$ expectations by denoting naive expectations as level-zero expecations and then defining level- $n$ expectations as the actual action of an opponent who has level- $n-1$ expectations. In the following two subsections we formalize this simple idea for expectation formation in the second and the first stage of the game.

\subsubsection{Stage two}

In case the expectations are formed at stage two, the actions in the first stage have been revealed and are taken into account when building the expectations We define the level- $n$ expectation generating functions in stage two recursively in the following way:

$\hat{q}_{j}^{0}\left(x_{1, t+1}, x_{2, t+1}, q_{1, t}, q_{2, t}\right)=q_{j, t}$

$\hat{q}_{j}^{n}\left(x_{1, t+1}, x_{2, t+1}, q_{1, t}, q_{2, t}\right)=R_{j}^{q}\left(\hat{q}_{i}^{n-1}\left(x_{1, t+1}, x_{2, t+1}, q_{1, t}, q_{2, t}\right) ; x_{1, t+1}, x_{2, t+1}\right), \quad i, j=1,2, i \neq j$ 
for all $\left(x_{1, t+1}, x_{2, t+1}, q_{1, t}, q_{2, t}\right) \in X_{1} \times X_{2} \times Q_{1} \times Q_{2}$. It should be noted that $\hat{q}_{j}^{n}$ does not depend on $q_{i, t}$ when $n$ is even and not on $q_{j, t}$ when $n$ is odd. For example for $n=2$ the expectation is formed from the perspective of player $i$, who expects that player $j$ anticipates that $q_{i, t+1}$ is chosen as the best response to the naive expectation $q_{j, t}$ and then chooses a best response to that anticipation about $q_{i, t+1}$. Hence, this expecation depends only on $q_{j, t}$, but not on $q_{i, t}$. To keep notation consistent we nevertheless write all expectations as functions of both stage two actions in the previous period.

Actual expectations in the second stage of period $t$ are then given by

$$
q_{j, t+1}^{2, n e}=\hat{q}_{j}^{n}\left(x_{1, t+1}, x_{2, t+1}, q_{1, t}, q_{2, t}\right)
$$

Clearly, for level $n=0$ players have naive expectations.

The two players choose their stage-two actions $\left(q_{1, t+1}, q_{2, t+1}\right)$ as best responses to these expectations. Hence, we have for a process of level- $n$

$$
q_{i, t+1}=R_{i}^{q}\left(\hat{q}_{j}^{n}\left(x_{1, t+1}, x_{2, t+1}, q_{1, t}, q_{2, t}\right), x_{1, t+1}, x_{2, t+1}\right) \quad i, j=1,2, \quad i \neq j,
$$

where $\hat{q}_{j}^{n}$ is given by (3.3). In order to determine the full dynamics of the system we have to move now to stage one in order to determine how $x_{1, t+1}, x_{2, t+1}$ are chosen.

\subsubsection{Stage one}

Expectation formation as well as the structure of the decision problem of the players in stage one differs in several respects from that in stage two. The main difference comes from the observation that the choice of $x_{i}$ in stage one influences the expectations of the opponent in stage two. The exact form of this influence depends on the level of the expectation process, which means that the shape of the best response functions of the players in stage one changes as the level of expectations changes. For a given (expected) value $x_{j, t+1}$ of the action of player $j$ in $t+1$ the problem of player $i$ in stage one reads:

$$
\begin{array}{r}
\max _{x_{i, t+1} \in X_{i}} \pi_{i}\left(x_{i, t+1}, x_{j, t+1}, R_{i}^{q}\left(\hat{q}_{j}^{n}\left(x_{1, t+1}, x_{2, t+1}, q_{1, t}, q_{2, t}\right), x_{i, t+1}, x_{j, t+1}\right),\right. \\
\left.\hat{q}_{j}^{n}\left(x_{1, t+1}, x_{2, t+1}, q_{1, t}, q_{2, t}\right)\right), \quad i, j=1,2, \quad i \neq j .
\end{array}
$$

The solution of this problem is referred to as the best response of player $i$ on stage one under level- $n$ expectations and denoted by $R_{i}^{x, n}\left(x_{j, t+1} ; q_{1, t}, q_{2, t}\right)$. Strictly speaking $n$ refers here to the level of expectation in stage two, since the best response function is independent from the way expectations are formed in stage one. In case the problem 
has several solutions an upper-hemi continuous selection is chosen. To understand the strategic effects emerging from the expectation formation it is instructive to consider the first order conditions of problem (3.5). It is given by ${ }^{2}$

$$
\frac{\partial \pi_{i}}{\partial x_{i, t+1}}+\frac{\partial \pi_{i}}{\partial q_{j}} \frac{\partial q_{j, t+1}^{n e}}{\partial x_{i, t+1}}=0 \quad i, j=1,2, \quad i \neq j
$$

where we have used the envelope theorem to drop the partial derivative of $\pi_{i}$ with respect to $q_{i}$ and have deleted the arguments in all functions for ease of notation. The second term in the sum captures the strategic effect emerging from the expectation formation on stage two and the sign and the size of this effect is driven by $\frac{\partial q_{j, t+1}^{n e}}{\partial x_{i, t+1}}$.

Having defined this best response function we can formulate expectations in stage one analogously to that in stage two. Since, the best response function in stage one depends on the level of expectations in stage two we have to recursively define the expectation generating functions in stage one for a given expectations level in stage two. In particular, we define the level- $m$ expectation generating function in stage one given that expectations are of level $n$ in stage two as:

$$
\begin{aligned}
\hat{x}_{j}^{0, n}\left(x_{1, t}, x_{2, t}, q_{1, t}, q_{2, t}\right) & =x_{j, t} \\
\hat{x}_{j}^{m, n}\left(x_{1, t}, x_{2, t}, q_{1, t}, q_{2, t}\right) & =R_{j}^{x, n}\left(\hat{x}_{i}^{m-1, n}\left(x_{1, t}, x_{2, t}, q_{1, t}, q_{2, t}\right) ; q_{1, t}, q_{2, t}\right) \quad i, j=1,2, i \neq j .
\end{aligned}
$$

for all $\left(x_{1, t}, x_{2, t}, q_{1, t}, q_{2, t}\right) \in X_{1} \times X_{2} \times Q_{1} \times Q_{2}$. To reduce notation we write $\hat{x}_{j}^{n, n}$ as $\hat{x}_{j}^{n}$. Similar to stage two expectations, $\hat{x}_{j}^{n}$ is independent from $x_{i, t}$ for $n$ even and independent from $x_{j, t}$ for $n$ odd. The level- $n$ expectations at stage one of period $t+1$ now read $x_{j, t+1}^{1, n e}=\hat{x}_{j}^{n}\left(x_{1, t}, x_{2, t}, q_{1, t}, q_{2, t}\right)$.

The resulting dynamics of stage-one actions under level $n$ expectations follow directly as

$$
x_{i, t+1}=R_{i}^{x, n}\left(\hat{x}_{j}^{n}\left(x_{1, t}, x_{2, t}, q_{1, t}, q_{2, t}\right) ; q_{1, t}, q_{2, t}\right) .
$$

Together with the dynamics of stage-two actions (3.4) this defines a dynamical system

$$
\left(x_{1, t+1}, x_{2, t+1}, q_{1, t+1}, q_{2, t+1}\right)=\Phi^{n}\left(\left(x_{1, t}, x_{2, t}, q_{1, t}, q_{2, t}\right)\right)
$$

with the generating map $\Phi^{n}: X_{1} \times X_{2} \times Q_{1} \times Q_{2} \mapsto X_{1} \times X_{2} \times Q_{1} \times Q_{2}$.

In the next section we will apply this general approach in order to formulate and analyze the expectations and the resulting dynamics of actions for different

\footnotetext{
${ }^{2}$ To keep things simple we assume here that $\hat{q}_{j}^{n}$ is differentiable at the considered point in the state space, which according to our assumptions holds true for points in the interior of the state space.
} 
expectation levels $n$ in the framework of a Cournot duopoly, where firms can invest in cost reducing activities before making their quantity choices. Before we turn to this application we formulate two general results characterizing the fixed points of the expectations dynamics for the extreme cases of the lowest level $n=0$ and $n$ going to infinity.

In the case of $n=0$, which corresponds to naive expectations, the fact that actions are taken sequentially and first stage actions are common knowledge in the second stage does not influence the long run outcomes of the dynamics (at least if the dynamics converges to a fixed point). The following Proposition shows that any fixed point of (3.9) corresponds to a Nash equilibrium of the game without revelation of action choices between the stages.

Proposition 3.1. Let $\left(\hat{x}_{1}, \hat{x}_{2}, \hat{q}_{1}, \hat{q}_{2}\right)$ be a fixed point of (3.9) for $n=0$. Then $\left(\hat{x}_{1}, \hat{x}_{2}, \hat{q}_{1}, \hat{q}_{2}\right)$ is a Nash-equilibrium of the game without revelation of actions between the stages.

It follows from standard results concerning best response dynamics with naive expectations in the literature that in the context of one-stage games the fixed points of these dynamics must be Nash equilibria of the game. Based on this, one implication of Proposition 3.1 is that under naive expectations the fixed points of the best response dynamics stay the same no matter whether the $x_{i}$ and $q_{i}$ are chosen simultaneusly or sequentially with full revelation of first-stage choices between the stages. This does not imply that the fact that choices are made sequentially is irrelevant for the shape of the trajectories of the dynamics. Actually, it is easy to see that in general the trajectories of(3.9) for $n=0$ differ from that of best response dynamics under naive expectations for the game where both players choose $x_{i}$ and $q_{i}$ in the same stage. The reason why the fixed points of the two dynamics nevertheless coincide is that under naive expectations expectations in stage two are completely unaffected by the choices in stage one (i.e. the second term in (A.11) is zero), which means that the best response function on stage one exactly coincides with the best response function for $x_{i}$ without revelation of choice between stages. Hence, the fixed points of stage-one actions in the two scenarios coincide. Since the best response functions on stage two (for given choices of $\left(x_{1}, x_{2}\right)$ ) also coincide the fact that the fixed points of stage one actions are the same implies that also the fixed points of stage-two actions have to coincide.

We now turn to the other extreme and analyze the fixed points of expectations dynamics if the expectations level is very high. Formally, we consider the limit $n \rightarrow \infty$. If the level of expectations is larger than zero, in general it is not ensured that players have correct expectations about the actions of the other player on stage 
one in every fixed point of the dynamics. There might be fixed points $\left(\hat{x}_{1}, \hat{x}_{2}, \hat{q}_{1}, \hat{q}_{2}\right)$ where players have expectations $\hat{x}_{i}^{1, n e} \neq \hat{x}_{i}$ with the property that $\hat{x}_{j}$ is a best response to $\hat{x}_{i}^{1, n e}$ and $\hat{x}_{i}^{1, n e}$ results from the application of the expectation generating function $\hat{x}_{i}^{n}$ to $\left(\hat{x}_{1}, \hat{x}_{2}, \hat{q}_{1}, \hat{q}_{2}\right)$. Such a phenomenon for example occurs for expectation level $n=1$ if the game without revelation of actions between stages has two Nash equilibria. Denoting the first-stage actions in the two equilibria by $\left(\bar{x}_{1}, \bar{x}_{2}\right)$ and $\left(\overline{\bar{x}}_{1}, \overline{\bar{x}}_{2}\right)$ with $\bar{x}_{i} \neq \overline{\bar{x}}_{i}, i=1,2$, it is easy to see that $\hat{x}_{1}=\bar{x}_{1}, \hat{x}_{2}=\overline{\bar{x}}_{2}$ with the associated expectations $\hat{x}_{1}^{1,1 e}=\overline{\bar{x}}_{1}, \hat{x}_{2}^{1,1 e}=\bar{x}_{2}$ is a fixed point of the expectations dynamics, but players do not have correct expectations and the fixed point does in general not correspond to a Nash equilibrium of the game. For levels $n>1$ even symmetric steady states of the dynamics with a symmetric underlying game might not be characterized by correct expectations, but might correspond to cycles of order $n$ of the stage-one best response functions $R_{i}^{x, n}$. In what follows we will however concentrate our attention to fixed points of the expectations dynamics, where expectations converge to the correct values. Formally we use the following definition.

Definition 3.2. A fixed point $\left(\hat{x}_{1}, \hat{x}_{2}, \hat{q}_{1}, \hat{q}_{2}\right)$ of the level-n expectations dynamics exhibits correct expectations in stage one if $\hat{x}_{i}=\hat{x}_{i}^{n}\left(\hat{x}_{1}, \hat{x}_{2}, \hat{q}_{1}, \hat{q}_{2}\right)$ for $i=1,2$.

A necessary condition to rule out any fixed points where players do not have correct expectation is that the best response functions on stage one are contraction mappings. Since we have assumed contraction properties for the best responses on stage two it is always guaranteed that expectations with respect to the actions on stage two are correct in a fixed point of the dynamics. Loosely speaking the following Proposition shows that the fixed points of (3.9) with correct expectations on stage one converge to subgame perfect equilibria of the game as the level of expectations becomes large.

Proposition 3.3. Let $\left(\hat{x}_{1}^{n}, \hat{x}_{2}^{n}, \hat{q}_{1}^{n}, \hat{q}_{2}^{n}\right)_{n=0}^{\infty}$ be a sequence of fixed points of (3.9) with correct expectations on stage one for increasing expectation levels $n$. Let $\left(\hat{x}_{1}^{*}, \hat{x}_{2}^{*}, \hat{q}_{1}^{*}, \hat{q}_{2}^{*}\right)$ be an interior accumulation point of that sequence. Then, $\left(\hat{x}_{1}^{*}, \hat{x}_{2}^{*}, \hat{q}_{1}^{*}, \hat{q}_{2}^{*}\right)$ is a subgameperfect equilibrium of the two-stage game.

Although our assumptions about the best responses on stage two guarantee a unique Nash equilibrium on stage two for any choice made on stage one, our framework allows for scenarios where the expectations dynamics has several fixed points with correct expectations for a given level $n$. According to Proposition 3.3 any limit point of sequences of such fixed points for $n \rightarrow \infty$ must be a subgame perfect equilibrium. Following our general assumption that the expectation levels are equal in 
both stages Proposition 3.3 is formulated for the case where levels in both stages tend to infinity. However, it should be noted that the Proposition also applies to all dynamics where the expectation level in the first stage is held constant at some arbitrary level, including naive expectations, and only the level of expectations about stage-two actions becomes large.Proposition 3.3 says that at least for large $n$ the fixed point of the dynamics is close to a subgame perfect equilibrium of the game. It is important to realize that Proposition 3.3 highlights a crucial difference between the properties of fixed points of expectations dynamics for one-stage and two-stage games. For one-stage games the set of fixed points with correct expectations is completely independent of the levels of expectations and only the speed of convergence, respectively the stability of the fixed points might depend on this parameter. For two-stage games the set of fixed points in general changes as the level of expectations is increased. This observation implies that the explicit consideration of the effects of expectation levels larger than one becomes much more crucial in the context of two-stage games. Whereas for one-stage games it can be loosely argued that higher levels in the long run lead to the same outcomes as level $n=0$ typically considered in the literature, for two-stage games this is no longer true. Therefore, it is a natural and relevant question to ask how 'more sophisticated' expectation formation in the sense of higher expectation levels influence the long run outcome of the dynamics. We will address these issues in the context of a two-stage duopoly model in the next section.

\section{Application to a Dynamic Duopoly with Cost Reducing Activities}

We now use the general framework developed in the previous section to study the dynamics of cost-reducing activities in a Cournot duopoly under different assumptions about expectation formation. In particular, we consider a dynamic version of a two-stage game introduced in Qiu (1997), where where firms produce horizontally differentiated goods in a Cournot Duopoly. In the first stage each firm undertakes cost-reducing R\&D efforts, and, in the second stage, the firms choose their quantities and produce. As discussed in the introduction the issue of strategic choice of cost-reducing activities in an oligopoly has attracted considerable attention in the industrial organization literature, and was analyzed both from a static and a dynamic perspective, where the cost reducing activities are typically interpreted as process innovation. We consider here cost reducing activities $(C R A)$ which are of a non-cumulative character. 
The inverse demand function for the product offered by firm $i$ is given by

$$
p_{i}=a-q_{i}-\gamma q_{j}, \quad i, j=1,2, \quad i \neq j
$$

with $\gamma \in(0,1)$. Due to cost reducing activities the firms can lower their marginal costs, given by

$$
c_{i}=c-x_{i}, \quad i=1,2, \quad x_{i} \in(0, c) .
$$

The $C R A$-expenditure function of firm $i$ is quadratic and of the form

$$
V\left(x_{i}\right)=\frac{v x_{i}^{2}}{2}, \quad i=1,2
$$

In terms of our general framework we have a game with strategy spaces $X_{i}=[0, \bar{X}]$, $Q_{i}=[0, a]$ for some large $\bar{X}$ and payoff functions

$$
\pi_{i}\left(x_{1}, x_{2}, q_{1}, q_{2}\right)=\left(a-q_{i}-\gamma q_{j}-c+x_{i}\right) q_{i}-V\left(x_{i}\right), \quad i, j=1,2, i \neq j .
$$

\subsection{Equilibrium Outcomes}

We first derive the subgame perfect equilibria of the game using backward induction. The chosen values of $x_{i}$ are revealed at the end of the first stage and become common knowledge. Given any first stage $C R A x_{1}, x_{2}$ the firms maximize the profit on the second stage with respect to the output. The first order condition $\frac{\partial \pi_{i}}{\partial q_{i}}=0$ gives rise to the best response function

$$
R_{i}^{q}\left(q_{j} ; x_{1}, x_{2}\right)=\operatorname{Max}\left[0, \frac{1}{2}\left(a-c+x_{i}-\gamma q_{j}\right)\right] .
$$

and the intersection of the two best response functions determines a unique CournotNash equilibrium, which in the case of positive equilibirum quantities is given by

$$
q_{i}^{*}\left(x_{1}, x_{2}\right)=\frac{(a-c)(2-\gamma)+2 x_{i}-\gamma x_{j}}{4-\gamma^{2}}, \quad i=1,2, \quad i \neq j
$$

Note that $\frac{\partial q_{i}^{*}}{\partial x_{i}}=\frac{2}{4-\gamma^{2}}>0$ and $\frac{\partial q_{j}^{*}}{\partial x_{i}}=-\frac{\gamma}{4-\gamma^{2}}<0$, i.e. the first-stage $C R A$ influences positively (negatively) the own (competitor's) output decision on the second stage and hence has an intertemporal strategic effect under the assumption that the Cournot equilibrium is realized in the second stage. However, the best response function of firm $i$ only depends on $x_{i}$, which means that for a given quantity of firm $j$ the best response of firm $i$ is independent from the first stage choice of the level of $C R A$ by firm $j$. 
Assuming that the induced quantities in the second stage are positive, in the first stage the firms maximize

$$
\pi_{i}^{*}\left(x_{i}\right)=\pi_{i}\left(x_{1}, x_{2}, q_{1}^{*}\left(x_{1}, x_{2}\right), q_{2}^{*}\left(x_{1}, x_{2}\right)\right)=\left(\frac{(a-c)(2-\gamma)+2 x_{i}-\gamma x_{j}}{4-\gamma^{2}}\right)^{2}-\frac{v}{2} x_{i}^{2},
$$

$i=1,2, i \neq j$ with respect to $x_{i}$. From the first order condition, (given in (A.2)) we obtain the following proposition.

Proposition 4.1. Assume that

$$
v> \begin{cases}\frac{4 a}{c\left(4-\gamma^{2}\right)(2+\gamma)} & \text { for } \frac{a}{c} \geqslant 2 \\ 1 & \text { for } 1<\frac{a}{c}<2\end{cases}
$$

Then, there exists a symmetric subgame perfect equilibrium with

$$
x_{1}^{*}=x_{2}^{*}=x_{S P}^{*}:=\frac{4(a-c)}{v(2-\gamma)(2+\gamma)^{2}-4} \in(0, c)
$$

and

$$
q_{1}^{*}=q_{2}^{*}=q_{S P}^{*}:=\frac{(a-c)(2-\gamma)+2 x_{i}^{*}-\gamma x_{j}^{*}}{4-\gamma^{2}}=\frac{v(a-c)\left(4-\gamma^{2}\right)}{v(2-\gamma)(2+\gamma)^{2}-4}>0 .
$$

The resulting equilibrium profit is given by

$$
\pi_{S P}^{*}=\left(q_{S P}^{*}\right)^{2}-\frac{v}{2}\left(x_{S P}^{*}\right)^{2}=\frac{v(a-c)^{2}\left(v\left(\gamma^{2}-4\right)^{2}-8\right)}{\left(4+v(\gamma-2)(2+\gamma)^{2}\right)^{2}} .
$$

The condition (4.2) guarantees that $\pi^{*}\left(x_{1}, x_{2}\right)$ is strictly concave with respect to $x_{i}$ on $X_{i}$.

In addition to this subgame perfect equilibrium the two-stage game has many Nash equilibria which are not subgame perfect. In general the set of Nash equilibrium outcomes includes all action profiles $\left(x_{1}, x_{2}, q_{1}, q_{2}\right)$ with $0<x_{i}<c$ and $q_{i}^{*}=q_{i}\left(x_{i}, x_{j}\right)$ for $i, j=1,2, i \neq j$. In particular we obtain:

Proposition 4.2. Define $\bar{x}=\min \left[\frac{(a-c)}{(2+\gamma) \sqrt{\frac{v}{2}}-1}, c\right]$ and consider an arbitrary $\tilde{x} \in$ $[0, \bar{x}]$. Then, there exists a symmetric Nash equilibrium $\left(x_{N E}, x_{N E}, q_{N E}(\cdot), q_{N E}(\cdot)\right)$ of the game, such that $x_{N E}=\tilde{x}$.

The level of $C R A$ 's denoted by $\bar{x}$ is the maximal level where firms have nonnegative marginal costs and non-negative profits in the corresponding Nash equilibrium. This Proposition shows that a large range of outcomes can be interpreted 
as a Nash equilibrium of the two stage game. In particular, under condition (4.2) any level of $C R A$ below that in the (unique) subgame perfect equilibrium is consistent with Nash equilibrium. If we consider a version of this game, where first stage actions are not revealed between the two stages, then the set of Nash equilibria collapses to a single point.

Proposition 4.3. Assume that $\left(x_{1}, x_{2}\right)$ is not revealed after the first stage and that $v>\frac{a}{(2+\gamma) c}$ and $a>c$ holds. Then, there exists a unique positive Nash equilibrium $\left(\widehat{x}_{1}, \widehat{x}_{2}, \widehat{q}_{1}, \widehat{q}_{2}\right)$ with

$$
\begin{aligned}
\widehat{x}_{1} & =\widehat{x}_{1}=\widehat{x}_{N E}:=\frac{a-c}{v(2+\gamma)-1} \in\left(0, x_{S P}^{*}\right) \text { and } \\
\widehat{q}_{1} & =\widehat{q}_{2}=\widehat{q}_{N E}:=\frac{v(a-c)}{v(2+\gamma)-1} \in\left(0, q_{S P}^{*}\right) .
\end{aligned}
$$

The profits of the two firms in that equilibrium is given by

$$
\widehat{\pi}_{N E}=\frac{v(2 v-1)(a-c)^{2}}{2(v(2+\gamma)-1)^{2}} .
$$

Comparing the unique subgame perfect equilibrium of the two-stage game with the unique Nash equilibrium of the game without revelation of stage-one choices, we realize that the revelation of stage 1 actions leads to an increase of the level of cost reducing activities and of quantities in equilibrium. Therefore, revelation of actions between the stages leads to lower prices, which implies a higher consumer surplus. On the other hand it can be verified that for all $\gamma \in[0,1], 0<c<a$ and all values of $v$ satisfying (4.2) the profit of the firms in the Nash equilibrium without revelation of stage 1 actions is larger compared to their profit in the subgame perfect equilibrium of the two-stage game. The economic intuition for these observations is that without revelation of the $C R A$ before the quantity choice of the opponent the additional incentives for $C R A$ s stemming from the negative effect a reduction of own costs on the opponents output disappears. Hence, firms invest less in CRA's in the game without revelation, which implies that marginal production costs are higher and quantities are lower. With respect to the firms' profits the positive effect of the increase of the opponents costs outweighs the own reduction in $C R A$ and therefore the profit is larger without revelation of stage 1 actions. The comparison of these two equilibria will turn out to be relevant for the understanding of the economic implications of an increase of the expectations level for the long run outcomes of the dynamics. We now turn to the analysis of these dynamics. 


\subsection{Level- $n$ expectations dynamics}

We assume now that firms repeatedly interact according to the two-stage game described above (with revelation of stage-one actions between the stages). Ex-ante they are not coordinated in one of the equilibria and they have to build expectations about the actions of the other firm in both stages. They build their expectations according to the expectations process with level $n$, as described in section 2 , and choose their actions as a best response to these expectations.

Like in the discussion of the general case, we start the analysis of the expectation dynamics by considering stage two. In that stage each firm has observed the level of cost reducing activities by the opponent and builds expectations according to (3.3). The following Lemma provides an explicit representation of the expectation generating function for level- $n$ expectations.

Lemma 4.4. The level $n$ expectation generating function on stage two concerning the quantity $q_{j, t+1}$ is given by

$$
\begin{aligned}
\hat{q}_{j}^{n}\left(x_{1, t+1}, x_{2, t+1}, q_{1, t}, q_{2, t}\right)= & \left(\frac{1}{2}\left(a-c+x_{j, t+1}\right)\right) \sum_{k=0}^{\lfloor(n-1) / 2\rfloor}\left(-\frac{\gamma}{2}\right)^{2 k} \\
& +\left(\frac{1}{2}\left(a-c+x_{i, t+1}\right)\right) \sum_{k=1}^{\lfloor n / 2\rfloor}\left(-\frac{\gamma}{2}\right)^{2 k-1}+\left(-\frac{\gamma}{2}\right)^{n} q_{\cdot, t},
\end{aligned}
$$

where $\lfloor u\rfloor$ denotes the largest integer smaller or equal than $u \in I R$ and $q_{\cdot, t}$ stands for $q_{i, t}$ if $n$ is odd and for $q_{j, t}$ if $n$ is even.

Considering the effect of the choice of cost reducing activities by firm $i$ in the first stage of $t+1$ on the expected quantity of firm $j$, it can be seen that an increase of the expectations level from $n$ to $n+1$ induces an increase of the coefficient of $x_{i, t+1}$ in $\hat{q}_{j}$ only if $n$ is odd. Put differently, if $n$ is even, then increasing the level by one does not change the size of the (negative) marginal effect of an increase of $x_{i, t+1}$ on the expected quantity of firm $j$ and therefore also the strategic incentive to invest stemming from the possibility to influence $q_{j, t+1}$ with $x_{i, t+1}$ should not change. Based on the explicit representation of the expectations in stage two the following Lemma gives the best response function in stage one under level- $n$ expectations.

Lemma 4.5. The best response function in stage one under level-n expectations in 
stage two for sufficiently large values of $(a-c)$ and $v>\frac{9}{8}$ is given by

$$
\begin{aligned}
& R_{i}^{x, n}\left(x_{j, t+1}, q_{1, t}, q_{2, t}\right) \\
& =\max \left[0, \frac{1+\sum_{k=1}^{\lfloor n / 2\rfloor}\left(-\frac{\gamma}{2}\right)^{2 k}}{2 v-\left(1+\sum_{k=1}^{\lfloor n / 2\rfloor}\left(-\frac{\gamma}{2}\right)^{2 k}\right)^{2}}\left((a-c) \sum_{k=0}^{n}\left(-\frac{\gamma}{2}\right)^{k}\right.\right. \\
& \left.\left.\quad-\gamma\left(-\frac{\gamma}{2}\right)^{n} q_{\cdot, t}+x_{j, t+1} \sum_{k=0}^{\lfloor(n-1) / 2\rfloor}\left(-\frac{\gamma}{2}\right)^{2 k+1}\right)\right],
\end{aligned}
$$

where $q_{\cdot, t}$ stands for $q_{i, t}$ if $n$ is odd and for $q_{j, t}$ if $n$ is even.

It should be noted that the coefficient of the $C R A$ of the opponent is negative, which means that, like in the static model, $C R A$ 's are strategic substitutes. Also it is easy to see that the absolute value of the coefficient of $x_{j, t+1}$ is smaller than 1 , which means that the best response function on stage one is a contraction mapping.

The expectations concerning the actions on stage one, $x_{j, t+1}^{1, n e}$ are built recursively according to (3.7) where the best response $R_{j}^{x, n}$ is given by (4.7). Since the resulting expression becomes rather large, we abstain from presenting the full expressions of these expectation functions here. It is easy to see that also these expectation functions are linear with respect to all arguments $x_{1, t}, x_{2, t}, q_{1, t}, q_{2, t}$. Using these specifications of the best response and expectation functions, the dynamics is given by (3.4) and (3.8).

Also for the fixed points of the expectations dynamics in principle a closed form expressions could be given. However, the complexity of these expressions would not allow substantial qualitative insights. Therefore, rather than providing these expressions we characterize in the following propositions several key properties of the sequence of the symmetric fixed points of the dynamics.

Proposition 4.6. Assume that $v>\frac{9}{8}$. Then there exists a unique symmetric interior fixed point $\left(\hat{x}^{n}, \hat{x}^{n}, \hat{q}^{n}, \hat{q}^{n}\right)$ of the level-n expectations dynamics. In the fixed point firms have correct expectations at stage one and the following properties hold:

(i) $\hat{x}^{0}=\hat{x}_{N E}, \hat{q}^{0}=\hat{q}_{N E}$

(ii) $\hat{x}^{n}=\hat{x}^{n+1}, \hat{q}^{n}=\hat{q}^{n+1}$ for $n$ even.

(iii) $\hat{x}^{n}<\hat{x}^{n+1}, \hat{q}^{n}<\hat{q}^{n+1}$ for $n$ odd.

(iv) $\lim _{n \rightarrow \infty} \hat{x}^{n}=x_{S P}^{*}, \lim _{n \rightarrow \infty} \hat{q}^{n}=q_{S P}^{*}$. 
Whereas items (i) and (iv) of the Proposition are just special cases of the general results given in Propositions 3.1 and 3.3, items (ii) and (iii) provide additional insights into the dependence of the fixed points on the level of expectations. In particular, the proposition shows that the level of $C R A$ 's and output in the fixed point increases monotonously as the level of expectations grows. However, if the considered level is even, then an increase by one does not alter the fixed point, so the increase happens 'stepwise' only at transitions from an odd to the next higher even level. As pointed out above, this is due to the fact that the best response on stage two is not directly influenced by the stage-one action of the other firm and therefore the size of the strategic effect of the stage one choice on the opponent's action on stage two changes only at every second increase of $n$. The observation that there are no fixed points of the dynamics where firms do not have correct expectations is due to the fact that the best response function on stage one is a contraction. This rules out multiple fixed points of the best response functions and best response cycles, which would be needed for fixed points where expectations are not correct.

Standard arguments imply that the characterized fixed points of the dynamics are asymptotically stable as long as $\gamma$ is sufficiently small. Precise conditions on this parameter guaranteeing stability are however rather involved and since the focus of the analysis is on the effect of the expectation level $n$ on the location of the fixed points we do not derive these conditions here. At the end of this section we will however briefly get back to the issue of stability of the fixed point.

From an economic perspective Proposition 4.6 shows that if firms increase the sophistication of their expectation rules, in the sense that they increase their expectation level, this leads in the long run to more cost reducing activities and higher outputs. As the expectation level $n$ goes up the fixed points move monotonously step-by-step from the Nash equilibrium of the game without revelation of cost reducing activities before the quantity choice towards the subgame perferct equilibrium of the two-stage game.

The effect of an increase in the expectation level is illustrated in Figure 4.1, where the size of $C R A$ 's and output quantities in the unique fixed point of the dynamics are depicted for different values of $n^{3}$ It can be clearly seen that for $n=0$ and $n=1$ the fixed point coincides with the Nash equilibrium of the game without revelation of actions between stages. However, already for $n=2$ firms reach in the long-run an outcome close to the subgame perfect equilibrium. As predicted in Proposition

\footnotetext{
${ }^{3}$ The parameter values used in all the figures are $a=5, c=2, \gamma=0.5, v=1.2$. Hence the conditions on $v$ of all Propositions in this section are satisfied.
} 


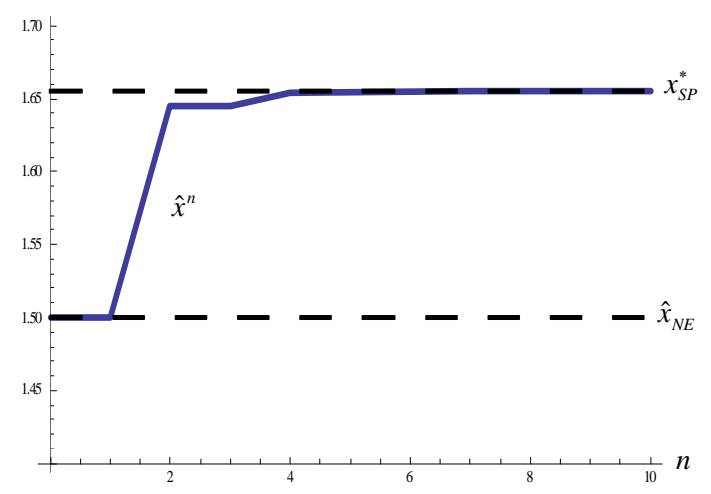

(a)

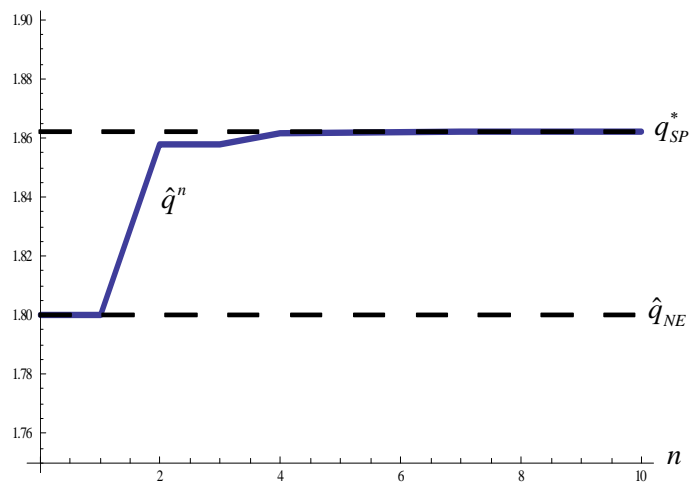

(b)

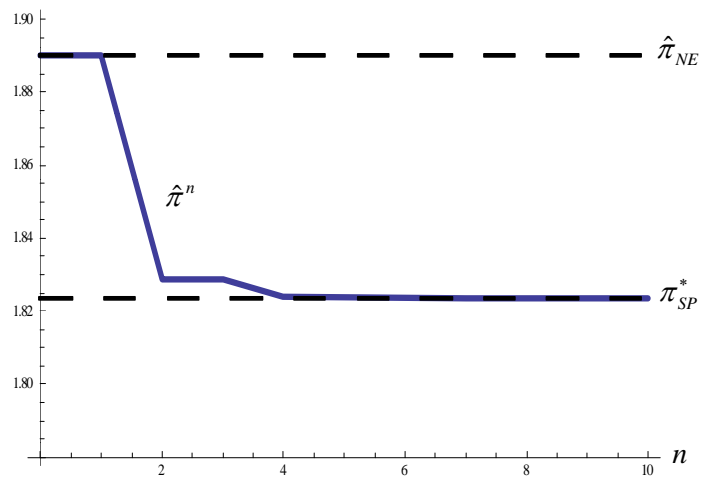

(c)

Figure 4.1: Level of cost reducing activities (a), output quantities (b) and firm profits (c) in the unique symmetric fixed point of the expectations dynamics for increasing $n$.

4.6 the long run outcome does not change if the expectation level is increased from $n=2$ to $n=3$. Moving to $n=4$ implies another small upward jump of long run $C R A$ 's and output of the firms. For this level the fixed point almost coincides with the subgame perfect equilibrium of the game. Therefore, further increases in the expectation level do not lead to any observable changes in the fixed point. Panel (c) of Figure 4.1 shows that this upwards movement of the fixed point values of $C R A$ 's and output associated with an increase in $n$ leads to a reduction in firms' profits. As discussed above, this is due to the fact that the negative price effect of the decrease of the opponent's marginal costs outweigh the positive profit effects of the increase in own $C R A$ 's. Increasing the level of sophistication in the expectation formation process of the firms in the industry therefore has negative implications for the average industry profits. Consumers however profit from such an increase in sophistication, since the market price goes downs as $n$ is increased.

The actual expectations dynamics for different expectation levels are illustrated 


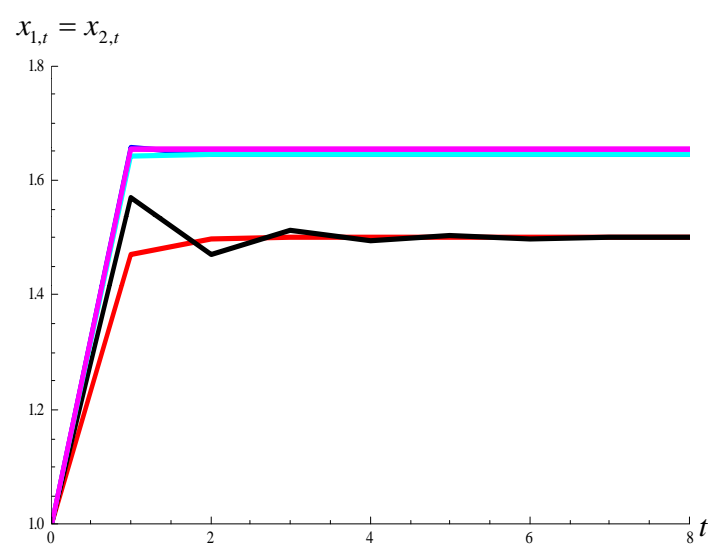

(a)

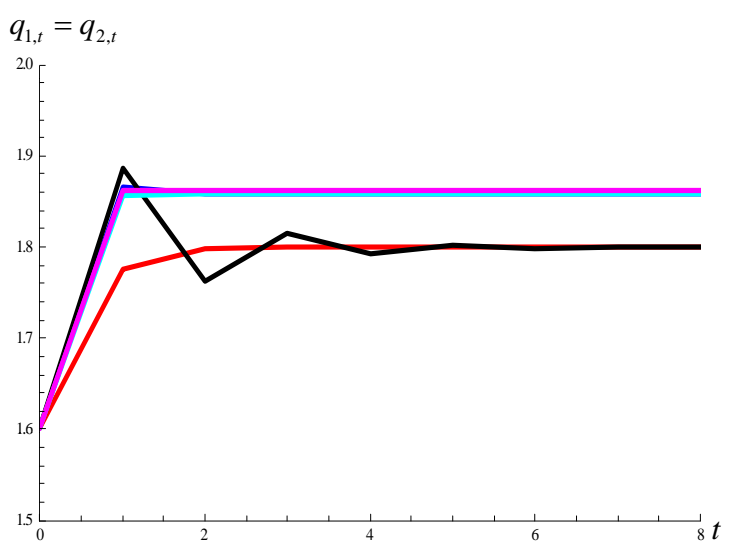

(b)

Figure 4.2: Dynamics of cost reducing activities (a) and output quantities (b) under different expectation levels: $n=0$ (black), 1 (red), 2 (dark blue), 3 (light blue), 4 (green) and 10 (pink).

in Figure 4.2. The figure shows that although the fixed points for $n=0$ and $n=1$ coincide the transient dynamics differ. In particular, under naive expectations some oscillations occur prior to convergence to the steady state, which are absent under expectations of level $n=1$. Starting with $n=2$ the dynamics look very similar, exhibiting very fast convergence to the steady state, which is close to the subgame perfect equilibrium of the game. Actually, due to the larger range of $x$ and $q$ values depicted in this figure compared to Figure 4.1 the difference in long run values between $n=2$ and larger levels is almost indiscernible. Overall, Figures 4.1 and 4.2 show that, whereas it is almost irrelevant whether naive expectations or expectations of level $n=1$ are considered, the transition to level $n=2$ leads to a significant qualitative change in transient and long run behavior. For such an expectation level firms reach after a few periods a state very close to the subgame perfect equilibrium of the game.

Although the focus of this paper is on the characterization of the fixed points of the expectation dynamics, we conclude the discussion by reconsidering the issue of stability of the fixed points. Figure 4.2 shows that for our default parameter setting, which includes a degree of horizontal product differentiation of $\gamma=0.5$ the fixed points of the dynamics are stable regardless of the expectation level. It is easy to see that decreasing the degree of vertical differentiation makes the best response functions on both stages steeper and therefore should contribute to a destabilization of the fixed point (see also Hadar (1966)). Based on this reasoning we show in Figure 4.3 the expectations dynamics for different expectation levels for the case $\gamma=0.95$, which means that the products of the two producers are close substitutes. It should 


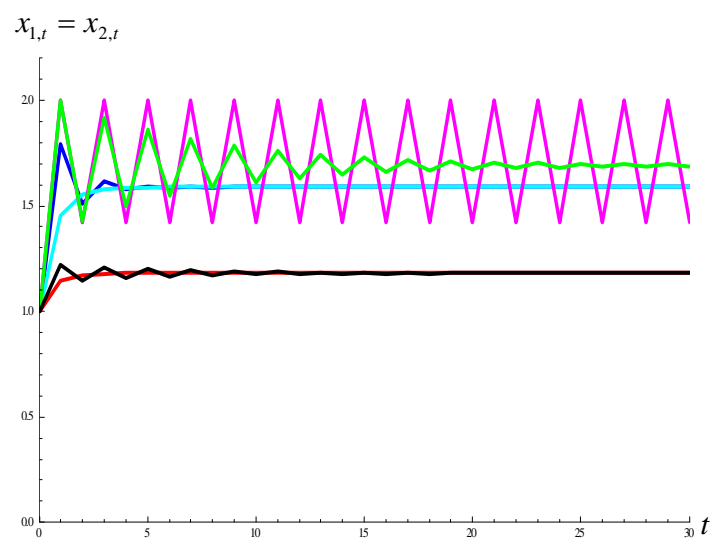

(a) $q_{1, t}=q_{2, t}$

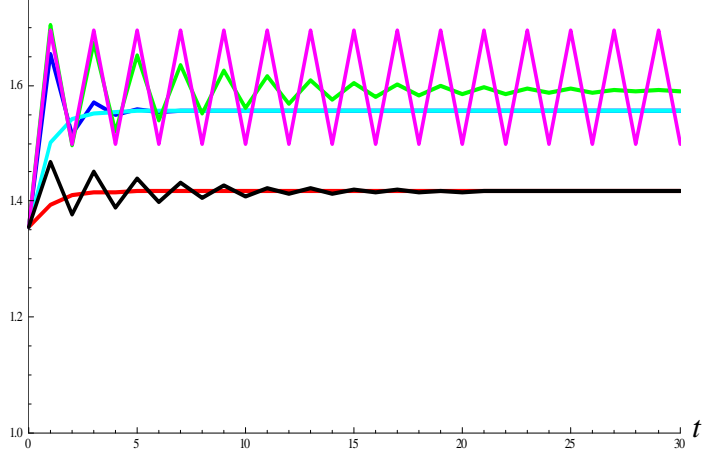

(b)

Figure 4.3: Dynamics of cost reducing activities (a) and output quantities (b) under level- $n$ expectations for $\gamma=.95: n=0$ (black), 1 (red), 2 (dark blue), 3 (light blue), 4 (green) and 10 (pink).

be noted that all conditions used in the Propositions of this section, in particular also the condition for the existence of an interior subgame perfect equilibrium are still satisfied for this parameter value. The destabilizing effect of the increase in $\gamma$ can be clearly seen. For expectation levels 0,2 and 4 both CRA's and quantities exhibit oscillations for several periods before they converge towards the steady state. For $n=10$ the fixed point now becomes unstable and $C R A$ 's and quantities end up in a period 2 cycle. This figure illustrates that more sophisticated expectation formation in the sense of a higher level of expectations might prevent firms from reaching a fixed point and coordinating in the long run. Whereas in the case of naive expectations or expectations with low level firms in the long have correct expectations, firms end up with persistently wrong expectations if their expectation level is large. This at first sight counter-intuitive finding is due to the fact that an increase in $n$ makes the negatively sloped best response function on stage one steeper (see (4.7)), which enlarges the danger of overshooting. 


\section{Conclusion}

This paper is a first contribution to systematically analyze the implications of different levels of expectations formation for best response dynamics in multistage games. Our analysis is focussed on a two-stage duopoly game where firms have sufficient information about the production technology of the opponent such that they can determine the best responses of the other firm on both stages. It is shown that in such a setting the long run outcome on the market depends crucially on the level of reasoning underlying the expectation formation process of the firms. An increase in this level does not lead to a smooth change of the trajectories. Rather, such an increase has no or very little effect for most transitions but leads to a rather aprupt change in the dynamics and long run outcome as the expectation level goes from $n=1$ to $n=2$. This means that whereas in the long run it does not make a difference whether firms use naive expectations or anticipate that the opponent will choose best responses to its naive expectations, there is a qualitative change in behavior if firms take into account that the opponent might go through similar reasoning than the firm itself and hold the expectation that about the firms own action that correspond to its best response. In the context of the considered two-class duopoly any more sophisticated reasoning than that does not lead a substantial change in the behavior. Although the long run outcome of (stable) best response dynamics depends on the level of expectation our results show that only outcomes between the Nash equilibrium of the game without revelation of actions between stage and the subgame perfect equilibrium of the game. Comparing this with the range of all Nash equilibrium outcomes shows that only a strict subset of Nash equilibria corresponds to fixed points of the best response dynamics under level- $n$ expectations. So, even without information about the actual level of expectations this dynamic approach provides some equilibrium selection.

In recent years substantial experimental work has been done to obtain a better understanding of how individuals build expectations in different market settings (e.g. Heemeijer et al. (2009), Hommes (2011)). The considered interaction structure in these experiments typically has one-stage strcuture. The insight obtained in this paper, that in two-stage problems market outcomes differ substantially depending on whether decision makers build level-1 or level-2 expectations might be a basis for experimental work on expectation formation in the framework of repeated two-stage market interaction.

The analysis in this paper has mainly focussed in the location of (stable) fixed points and has dealt with a linear-quadratic duopoly model with a unique subgameperfect equilibrium. As has been documented in the literature considering nonlinear 
demand or cost specifications would certainly give rise to co-existence of locally stable fixed points, or more generally attractors, and complex dynamical patterns. A rigorous analysis how basins of attraction or dynamic patterns in standard two-stage oligopoly games are affected by the level of expectation formation is an interesting challenge for future work. 


\section{References}

Bischi, G.-I., C. Chiarella, M. Kopel, and R. Szidarovszky (2010): Nonlinear Oligopolies, Springer.

Bischi, G.-I. AND M. Kopel (2001): "Equilibrium selection in a nonlinear duopoly game with adaptive expectations," Journal of Economic Behavior $\&$ Organization, 46, 73-100.

Bischi, G.-I. And F. Lamantia (2004): "A competition game with knowledge accumulation and spillovers," International Game Theory Review, 6, 323-241.

Breton, M., A. Turki, And G. Zaccour (2004): "Dynamic model of R\&D, spillovers, and efficiency of bertrand and cournot equilibria," Journal of Optimization Theory and Applications, 23, 1-25.

Brown, G. (1951): "Iterative Solutions of Games by Fictitious Play," in Activity Analysis of Production and Allocation, ed. by T. Koopmans, Wiley: New York.

Cellini, R. And L. Lambertini (2009): "Dynamic R\&D with spillovers: competition versus cooperation," Journal of Economic Dynamics and Control, 33, $568-582$.

- (2011): "R\&D incentives under Bertrand competition: a differential game," The Japanese Economic Review, 62, 387-400.

Cressman, R. And K. Schlag (1998): "The dynamic (in)stability of backwards induction," Journal of Economic Theory, 83, 260-285.

D'Aspremont, C. And A. JaCQuemin (1988): "Cooperative and noncooperative R\&D in duopoly with spillovers," American Economic Review, 78, 1133-1137.

Demichelis, S. And K. Ritzberger (2003): "From evolutionary to strategic stability," Journal of Economic Theory, 113, 51-75.

HADAR, J. (1966): "Stability of Oligopoly with Product Differentiation," The Review of Economic Studies, 33, 57-60.

HART, S. (2002): "Evolutionary dynamics and backward induction," Games and Economic Behavior, 41, 227-264.

Heemeijer, P., C. Hommes, J. Sonnemans, and J. Tuinstra (2009): "Price stability and volatility in markets with positive and negative expectations feedback," Journal of Economic Dynamics and Control, 33, 1052-1072. 
Hommes, C. (2011): "The heterogeneous expectations hypothesis: Some evidence from the lab," Journal of Economic Dynamics and Control, 35, 1-24.

Kopel, M. (2009): "Oligopoly Dynamics," in Handbook of Research on Complexity, ed. by J. J.B. Rosser, Edward Elgar.

Kuzmics, C. (2004): "Stochastic evolutionary stability in extensive form games of perfect information," Games and Economic Behavior, 48, 321-336.

Noeldecke, G. And L. SAmuelson (1993): "An evolutionary analysis of backward and forward induction," Games and Economic Behavior, 5, 425-454.

Okuguchi, K. (1970): "Expectations in an Oligopoly Model," Review of Economic Studies, 37, 233-237.

PuU, T. (1998): "The chaotic Duopolists revisited," Journal of Economic Behavior \& Organization, 33, 385-394.

QIU, L. D. (1997): "On the Dynamic Efficiency of Bertrand and Cournot Equilibria," Journal of Economic Theory, 75, 213-229.

Stahl, D. And P. Wilson (1995): "On players' models of other players: Theory and experimental evidence," Games and Economic Behavior, 10, 218-254.

Theocharis, R. (1960): "On the Stability of the Cournot Solution on the Oligopoly Problem," The Review of Economic Studies, 27, 133-134.

Tramontana, F., L. Gardini, And T. PuU (2009): "Cournot duopoly when competitors operate multiple production plants," Journal of Economic Dynamics and Control, 33, 250-265. 


\section{A Proofs}

\section{Proof of Proposition 3.1}

The fact that $\left(\hat{x}_{1}, \hat{x}_{2}, \hat{q}_{1}, \hat{q}_{2}\right)$ is a fixed point of (3.9) and expectations are naive implies using (3.4) and (3.8) that

$$
\hat{q}_{i}=R_{i}^{q}\left(\hat{q}_{j}, \hat{x}_{1}, \hat{x}_{2}\right), \quad i=1,2
$$

and

$$
\hat{x}_{i}=R_{i}^{x, 0}\left(\hat{x}_{j} ; \hat{x}_{1}, \hat{x}_{2}, \hat{q}_{1}, \hat{q}_{2}\right), \quad i=1,2
$$

hold. From (A.1) we directly obtain that $\hat{q}_{i}=q_{i}^{*}\left(\hat{x}_{1}, \hat{x}_{2}\right)$ and (3.5) yields under consideration of $q_{j, t+1}^{0 e}=q_{j, t}=\hat{q}_{j}$ that

$$
R_{i}^{x 0}\left(\hat{x}_{j} ; \hat{x}_{1}, \hat{x}_{2}, \hat{q}_{1}, \hat{q}_{2}\right)=\arg \max _{x_{i} \in X_{i}} \pi_{i}\left(x_{i}, \hat{x}_{j}, R_{i}^{q}\left(\hat{q}_{j}, x_{i}, \hat{x}_{j}\right), \hat{q}_{j}\right)
$$

Using (3.2) this implies

$$
\hat{x}_{i}=R_{i}^{x 0}\left(\hat{x}_{j} ; \hat{x}_{1}, \hat{x}_{2}, \hat{q}_{1}, \hat{q}_{2}\right)=\hat{R}_{i}^{x}\left(\hat{x}_{j}, \hat{q}_{j}\right)
$$

Accoringly, $\left(\hat{x}_{1}, \hat{x}_{2}, \hat{q}_{1}, \hat{q}_{2}\right)$ satsifies (3.1) and therefore is a Nash equilibrium of the game without revelation of action choices between the stages.

Q.E.D.

\section{Proof of Proposition 3.3}

First, it should be noted that due to our assumption that $\pi_{i}^{*}\left(x_{1}, x_{2}\right)$ is strictly convex with respect to $x_{i}$, the first order condition

$\frac{\partial \pi_{i}^{*}\left(\hat{x}_{1}^{*}, \hat{x}_{2}^{*}\right)}{\partial x_{i}}=\frac{\partial \pi_{i}\left(\hat{x}_{1}^{*}, \hat{x}_{2}^{*}, \hat{q}_{1}^{*}, \hat{q}_{2}^{*}\right)}{\partial x_{i}}+\frac{\partial \pi_{i}\left(\hat{x}_{1}^{*}, \hat{x}_{2}^{*}, \hat{q}_{1}^{*}, \hat{q}_{2}^{*}\right)}{\partial q_{j}} \frac{\partial q_{j}^{*}\left(\hat{x}_{1}, \hat{x}_{2}\right)}{\partial x_{i}}=0, i, j=1,2, i \neq j$

in combination with

$$
\hat{q}_{i}^{*}=q_{i}^{*}\left(\hat{x}_{1}^{*}, \hat{x}_{2}^{*}\right), i=1,2
$$

is a sufficient condition for an interior point $\left(\hat{x}_{1}^{*}, \hat{x}_{2}^{*}, \hat{q}_{1}^{*}, \hat{q}_{2}^{*}\right)$ to be a subgame-perfect equilibrium of the game. Since $\left(\hat{x}_{1}^{*}, \hat{x}_{2}^{*}, \hat{q}_{1}^{*}, \hat{q}_{2}^{*}\right)$ is an interior accumulation point of the sequence $\left(\hat{x}_{1}^{n}, \hat{x}_{2}^{n}, \hat{q}_{1}^{n}, \hat{q}_{2}^{n}\right)_{n=0}^{\infty}$ there must exist an interior subsequence $\left(n_{k}\right)_{k=0}^{\infty}$ with $\lim _{k \rightarrow \infty}\left(\hat{x}_{1}^{n_{k}}, \hat{x}_{2}^{n_{k}}, \hat{q}_{1}^{n_{k}}, \hat{q}_{2}^{n_{k}}\right)=\left(\hat{x}_{1}^{*}, \hat{x}_{2}^{*}, \hat{q}_{1}^{*}, \hat{q}_{2}^{*}\right)$. To reduce notation we assume without restriction of generality that the original sequence already has these properties.

To reduce notation we define $\hat{q}_{i}^{2, n e}=q_{i}^{2, n e}\left(\hat{x}_{1}^{n}, \hat{x}_{2}^{n}, \hat{q}_{1}^{n}, \hat{q}_{2}^{n}\right)$ for $i=1,2$. Because $\left(\hat{x}_{1}^{n}, \hat{x}_{2}^{n}, \hat{q}_{1}^{n}, \hat{q}_{2}^{n}\right)$ is a fixed point of $(3.9)$ with $\alpha=0$ we obtain from (3.4) that $\hat{q}_{i}=$ 
$R_{i}^{q}\left(\hat{q}_{j}^{2, n e} ; \hat{x}_{1}^{n}, \hat{x}_{2}^{n}\right), i, j=1,2, i \neq j$. Furthermore, assuming that $n$ is odd, it follows from (3.3) that

$$
\hat{q}_{j}^{2, n e}=R_{j}^{q}(\underbrace{R_{i}^{q}\left(R_{j}^{q}\left(\ldots R_{i}^{q}\left(R_{j}^{q}\left(\hat{q}_{i} ; \hat{x}_{1}^{n}, \hat{x}_{2}^{n}\right) ; \hat{x}_{1}^{n}, \hat{x}_{2}^{n}\right) ; \ldots ; \hat{x}_{1}^{n}, \hat{x}_{2}^{n}\right) ; \hat{x}_{1}^{n}, \hat{x}_{2}^{n}\right)}_{(n-1) / 2 \text { times }} ; \hat{x}_{1}^{n}, \hat{x}_{2}^{n})
$$

Together with $\hat{q}_{i}=R_{i}^{q}\left(\hat{q}_{j}^{2, n e} ; \hat{x}_{1}^{n}, \hat{x}_{j}^{n}\right)$ this implies that $\hat{q}_{i}$ is a fixed point of the $n / 2+1$ times composition of the function $R_{i}^{q}\left(R_{j}^{q}\left(\phi ; \hat{x}_{1}^{n}, \hat{x}_{2}^{n}\right) ; \hat{x}_{1}^{n}, \hat{x}_{2}^{n}\right)$. Since this function is assumed to be a contraction, also the $n / 2+1$ times composition is a contraction and therefore has a unique fixed point in $Q_{i}$. Therefore, the fixed point of this composition must coincide with the fixed point of $R_{i}^{q}\left(R_{j}^{q}\left(\phi ; \hat{x}_{1}^{n}, \hat{x}_{2}^{n}\right) ; \hat{x}_{1}^{n}, \hat{x}_{2}^{n}\right)$ and we obtain that $\hat{q}_{i}^{n}=q_{i}^{*}\left(\hat{x}_{1}^{n}, \hat{x}_{2}^{n}\right), i=1,2$. Analogous arguments establish that this property also must hold if $n$ is even. Since $q_{i}^{*}$ is continuous this implies that (A.3) holds.

It remains to be shown that (A.2) holds true. Due to our assumption that players have rational expectations on stage 1 in the considered fixed points we must have $\hat{x}_{i}^{n}=R_{i}^{x, n}\left(R_{j}^{x, n}\left(\hat{x}_{i} ; \hat{q}_{1}^{n}, \hat{q}_{2}^{n}\right) ; \hat{q}_{1}^{n}, \hat{q}_{2}^{n}\right)=R_{i}^{x, n}\left(\hat{x}_{j}^{n} ; \hat{q}_{1}^{n}, \hat{q}_{2}^{n}\right)$. Comparing the corresponding first order condition

$$
\frac{\partial \pi_{i}\left(\hat{x}_{1}^{n}, \hat{x}_{2}^{n}, \hat{q}_{1}^{n}, \hat{q}_{2}^{n}\right)}{\partial x_{i}}+\frac{\partial \pi_{i}\left(\hat{x}_{1}^{n}, \hat{x}_{2}^{n}, \hat{q}_{1}^{n}, \hat{q}_{2}^{n}\right)}{\partial q_{j}} \frac{\partial q_{j, t+1}^{n e}\left(\hat{x}_{1}^{n}, \hat{x}_{2}^{n}, \hat{q}_{1}^{n}, \hat{q}_{2}^{n}\right)}{\partial x_{i}}=0
$$

with (A.2), it becomes clear that all which remains to be shown is that

$$
\lim _{n \rightarrow \infty} \frac{\partial q_{j, t+1}^{n e}\left(\hat{x}_{1}^{n}, \hat{x}_{2}^{n}, \hat{q}_{1}^{n}, \hat{q}_{2}^{n}\right)}{\partial x_{i}}=\frac{\partial q_{j}^{*}\left(\hat{x}_{1}, \hat{x}_{2}\right)}{\partial x_{i}}
$$

holds. Considering this partial derivative we first observe that

$$
\frac{\partial q_{j, t+1}^{n e}\left(\hat{x}_{1}^{n}, \hat{x}_{2}^{n}, \hat{q}_{1}^{n}, \hat{q}_{2}^{n}\right)}{\partial x_{i}}=\frac{\partial \hat{q}_{j}^{n}\left(\hat{x}_{1}^{n}, \hat{x}_{2}^{n}, \hat{q}_{1}^{n}, \hat{q}_{2}^{n}, \hat{q}^{n}\right)}{\partial x_{i}},
$$

where $\hat{q}^{n}$ stands for $\hat{q}_{i}^{n}$ or $\hat{q}_{j}^{n}$ depending on whether $n$ is odd or even. The recursive definition of $\hat{q}_{j}^{n}$ induces the following recursion for this partial derivative:

$$
\begin{aligned}
& \frac{\partial \hat{q}_{j}^{n}\left(\hat{x}_{1}^{n}, \hat{x}_{2}^{n}, \hat{q}_{1}^{n}, \hat{q}_{2}^{n}, \hat{q}^{n}\right)}{\partial x_{i}}= \\
& \begin{cases}\frac{\partial \hat{q}_{j}^{n-1}\left(\hat{x}_{1}^{n}, \hat{x}_{2}^{n}, \hat{q}_{1}^{n}, \hat{q}_{2}^{n}, \hat{q}^{n}\right)}{\partial x_{i}}+\frac{\partial \hat{q}_{j}^{n-1}\left(\hat{x}_{1}^{n}, \hat{x}_{2}^{n}, \hat{q}_{1}^{n}, \hat{q}_{2}^{n}, \hat{q}^{n}\right)}{\partial q .} \frac{\partial R_{i}^{q}\left(\hat{q}_{j}^{n}, \hat{x}_{1}^{n}, \hat{x}_{2}^{n}\right)}{\partial x_{i}} & n \text { even } \\
\frac{\partial \hat{q}_{j}^{n-1}\left(\hat{x}_{1}^{n}, \hat{x}_{2}^{n}, \hat{q}_{1}^{n}, \hat{q}_{2}^{n}, \hat{q}_{.}^{n}\right)}{\partial x_{i}}+\frac{\partial \hat{q}_{j}^{n-1}\left(\hat{x}_{1}^{n}, \hat{x}_{2}^{n}, \hat{q}_{1}^{n}, \hat{q}_{2}^{n}, \hat{q}^{n}\right)}{\partial q .} \frac{\partial R_{j}^{q}\left(\hat{q}_{i}^{n}, \hat{x}_{1}^{n}, \hat{x}_{2}^{n}\right)}{\partial x_{i}} & n \text { odd },\end{cases}
\end{aligned}
$$


where $\frac{\partial \hat{q}_{j}^{n}\left(\hat{x}_{1}^{n}, \hat{x}_{2}^{n}, \hat{q}_{1}^{n}, \hat{q}_{2}^{n}, \hat{q}_{.}^{n}\right)}{\partial q .}$ follows the recursion

$$
\frac{\partial \hat{q}_{j}^{n}\left(\hat{x}_{1}^{n}, \hat{x}_{2}^{n}, \hat{q}_{1}^{n}, \hat{q}_{2}^{n}, \hat{q}^{n}\right)}{\partial q .}= \begin{cases}\frac{\partial \hat{q}_{j}^{n-1}\left(\hat{x}_{1}^{n}, \hat{x}_{2}^{n}, \hat{q}_{1}^{n}, \hat{q}_{2}^{n}, \hat{q}^{n}\right)}{\partial q .} \frac{\partial R_{j}^{q}\left(\hat{q}_{j}^{n} ; \hat{x}_{1}^{n}, \hat{x}_{2}^{n}\right)}{\partial q_{i}} & n \text { even } \\ \frac{\partial \hat{q}_{j}^{n-1}\left(\hat{x}_{1}^{n}, \hat{x}_{2}^{n}, \hat{q}_{1}^{n}, \hat{q}_{2}^{n}, \hat{q}^{n}\right)}{\partial q .} \frac{\partial R_{i}^{q}\left(\hat{q}_{j}^{n} ; \hat{x}_{1}^{n}, \hat{x}_{2}^{n}\right)}{\partial q_{j}} & n \text { odd } .\end{cases}
$$

Furthermore, we have

$$
\frac{\partial \hat{q}_{j}^{0}\left(\hat{x}_{1}^{n}, \hat{x}_{2}^{n}, \hat{q}_{1}^{n}, \hat{q}_{2}^{n}, \hat{q}_{.}^{n}\right)}{\partial x_{i}}=0, \quad \frac{\partial \hat{q}_{j}^{0}\left(\hat{x}_{1}^{n}, \hat{x}_{2}^{n}, \hat{q}_{1}^{n}, \hat{q}_{2}^{n}, \hat{q}_{.}^{n}\right)}{\partial q}=1 .
$$

for $i, j=1,2$ and $i \neq j$. Direct calculation based on these expressions establishes that the partial derivative with resepect to the last argument $q$. of the expectation generating function at stage 2 is given by

$$
\frac{\partial \hat{q}_{j}^{n}\left(\hat{x}_{1}^{n}, \hat{x}_{2}^{n}, \hat{q}_{1}^{n}, \hat{q}_{2}^{n}, \hat{q}^{n}\right)}{\partial q .}= \begin{cases}\left(\frac{\partial R_{i}^{q}\left(\hat{q}_{j} ; \hat{x}_{1}, \hat{x}_{2}\right)}{\partial q_{j}}\right)^{n / 2}\left(\frac{\partial R_{j}^{q}\left(\hat{q}_{i} ; \hat{x}_{1}, \hat{x}_{2}\right)}{\partial q_{i}}\right)^{n / 2} & n \text { even } \\ \left(\frac{\partial R_{i}^{q}\left(\hat{q}_{j} ; \hat{x}_{1}, \hat{x}_{2}\right)}{\partial q_{j}}\right)^{(n-1) / 2}\left(\frac{\partial R_{j}^{q}\left(\hat{q}_{i} ; \hat{x}_{1}, \hat{x}_{2}\right)}{\partial q_{i}}\right)^{(n+1) / 2} & n \text { odd }\end{cases}
$$

Inserting this into (A.4) finally yields

$$
\begin{aligned}
& \frac{\partial \hat{q}_{j}^{n}\left(\hat{x}_{1}^{n}, \hat{x}_{2}^{n}, \hat{q}_{1}^{n}, \hat{q}_{2}^{n}, \hat{q}_{.}^{n}\right)}{\partial x_{i}}= \\
& \begin{cases}\frac{\partial R_{i}^{q}}{\partial x_{i}} \sum_{k=1}^{n / 2}\left(\frac{\partial R_{i}^{q}}{\partial q_{j}}\right)^{k-1}\left(\frac{\partial R_{j}^{q}}{\partial q_{i}}\right)^{k}+\frac{\partial R_{j}^{q}}{\partial x_{i}} \sum_{k=0}^{n / 2-1}\left(\frac{\partial R_{i}^{q}}{\partial q_{j}}\right)^{k}\left(\frac{\partial R_{j}^{q}}{\partial q_{i}}\right)^{k} & n \text { even } \\
\frac{\partial R_{i}^{q}}{\partial x_{i}} \sum_{k=1}^{(n-1) / 2}\left(\frac{\partial R_{i}^{q}}{\partial q_{j}}\right)^{k-1}\left(\frac{\partial R_{j}^{q}}{\partial q_{i}}\right)^{k}+\frac{\partial R_{j}^{q}}{\partial x_{i}} \sum_{k=0}^{(n-1) / 2}\left(\frac{\partial R_{i}^{q}}{\partial q_{j}}\right)^{k}\left(\frac{\partial R_{j}^{q}}{\partial q_{i}}\right)^{k} & n \text { odd },\end{cases}
\end{aligned}
$$

where we again have dropped all functional arguments on the right side to lighten the notation. Due to our assumption that the composition of the two best response functions on stage 2 is a contraction we have $\left\|\frac{\partial R_{i}^{q}}{\partial q_{j}} \frac{\partial R_{j}^{q}}{\partial q_{i}}\right\|_{\infty}<1$ and taking the limit for $n \rightarrow \infty$ in (A.5) we obtain

$$
\begin{gathered}
\lim _{n \rightarrow \infty} \frac{\partial q_{j, t+1}^{2, n e}\left(\hat{x}_{1}^{n}, \hat{x}_{2}^{n}, \hat{q}_{1}^{n}, \hat{q}_{2}^{n}\right)}{\partial x_{i}}=\lim _{n \rightarrow \infty} \frac{\partial \hat{q}_{j}^{n}\left(\hat{x}_{1}^{n}, \hat{x}_{2}^{n}, \hat{q}_{1}^{n}, \hat{q}_{2}^{n}, \hat{q}^{n}\right)}{\partial x_{i}}= \\
\frac{\left(\frac{\partial R_{i}^{q}\left(q_{j}^{*} ; x_{1}^{*}, x_{2}^{*}\right)}{\partial x_{i}}\right)\left(\frac{\partial R_{j}^{q}\left(q_{i}^{*} ; x_{1}^{*}, x_{2}^{*}\right)}{\partial q_{j}}\right)+\frac{\partial R_{j}^{q}\left(q_{i}^{*} ; x_{1}^{*}, x_{2}^{*}\right)}{\partial x_{i}}}{1-\left(\frac{\partial R_{i}^{q}\left(q_{j}^{*} ; x_{1}^{*}, x_{2}^{*}\right)}{\partial q_{j}}\right)\left(\frac{\partial R_{j}^{q}\left(q_{i}^{*} ; x_{1}^{*}, x_{2}^{*}\right)}{\partial q_{j}}\right)} .
\end{gathered}
$$

To conclude the proof we have to compare this expression to $\frac{\partial q_{j}^{*}\left(\hat{x}_{1}^{*}, \hat{x}_{2}^{*}\right)}{\partial x_{i}}$. Since $q_{j}^{*}$ gives 
the Nash equilibrium choice of player $j$ on stage 2 it has to satisfy

$$
q_{j}^{*}\left(x_{1}, x_{2}\right)=R_{j}^{q}\left(R_{i}^{q}\left(q_{j}^{*}\left(x_{1}, x_{2}\right) ; x_{1}, x_{2}\right) ; x_{1}, x_{2}\right)
$$

for all $\left(x_{1}, x_{2}\right) \in X_{1} \times X_{2}$. Taking the derivative with respect to $x_{i}$ on both sides at $\left(x_{1}, x_{2}\right)=\left(\hat{x}_{1}^{*}, \hat{x}_{2}^{*}\right)$ yields

$$
\frac{\partial q_{j}^{*}\left(\hat{x}_{1}^{*}, \hat{x}_{2}^{*}\right)}{\partial x_{i}}=\frac{\partial R_{j}^{q}\left(\hat{q}_{i}^{*} ; \hat{x}_{1}^{*}, \hat{x}_{2}^{*}\right)}{\partial x_{i}}+\frac{\partial R_{j}^{q}\left(\hat{q}_{i}^{*} ; \hat{x}_{1}^{*}, \hat{x}_{2}^{*}\right)}{\partial q_{i}}\left(\frac{\partial R_{i}^{q}\left(\hat{q}_{j}^{*} ; \hat{x}_{1}^{*}, \hat{x}_{2}^{*}\right)}{\partial x_{i}}+\frac{\partial R_{i}^{q}\left(\hat{q}_{j}^{*} ; \hat{x}_{1}^{*}, \hat{x}_{2}^{*}\right)}{\partial q_{j}} \frac{\partial q_{j}^{*}\left(\hat{x}_{1}^{*}, \hat{x}_{2}^{*}\right)}{\partial x_{i}}\right),
$$

where we have used that $\hat{q}_{j}^{*}=q_{j}^{*}\left(\hat{x}_{1}^{*}, \hat{x}_{2}^{*}\right)$ and $\hat{q}_{i}^{*}=R_{i}^{q}\left(\hat{q}_{j}^{*} ; \hat{x}_{1}^{*}, \hat{x}_{2}^{*}\right)$. Collecting terms gives

$$
\frac{\partial q_{j}^{*}\left(\hat{x}_{1}^{*}, \hat{x}_{2}^{*}\right)}{\partial x_{i}}=\frac{\frac{\partial R_{j}^{q}\left(\hat{q}_{i}^{*} ; \hat{x}_{1}^{*}, \hat{x}_{2}^{*}\right)}{\partial x_{i}}+\left(\frac{\partial R_{j}^{q}\left(\hat{q}_{i}^{*} ; \hat{x}_{1}^{*}, \hat{x}_{2}^{*}\right)}{\partial q_{i}}\right)\left(\frac{\partial R_{i}^{q}\left(\hat{q}_{j}^{*} ; \hat{x}_{1}^{*}, \hat{x}_{2}^{*}\right)}{\partial x_{i}}\right)}{1-\left(\frac{\partial R_{j}^{q}\left(\hat{q}_{i}^{*} ; \hat{x}_{1}^{*}, \hat{x}_{2}^{*}\right)}{\partial q_{i}}\right)\left(\frac{\partial R_{i}^{q}\left(\hat{q}_{j}^{*} ; \hat{x}_{1}^{*}, \hat{x}_{2}^{*}\right)}{\partial q_{j}}\right)}
$$

and we have shown that

$$
\lim _{n \rightarrow \infty} \frac{\partial q_{j, t+1}^{2, n e}\left(\hat{x}_{1}^{n}, \hat{x}_{2}^{n}, \hat{q}_{1}^{n}, \hat{q}_{2}^{n}\right)}{\partial x_{i}}=\frac{\partial q_{j}^{*}\left(\hat{x}_{1}^{*}, \hat{x}_{2}^{*}\right)}{\partial x_{i}}
$$

This concludes the proof.

Q.E.D.

\section{Proof of Proposition 4.1:}

Direct calculations give the first order condition

$$
\frac{\partial \pi_{i}^{*}\left(x_{1}, x_{2}\right)}{\partial x_{i}}=\frac{2}{4-\gamma^{2}}\left(a-c+x_{i}^{*}-\gamma q_{j}^{*}\right)-v x_{i}^{*}=0
$$

In order to guanrantee that the first order condition characterizes the maximum of $p i^{*}$ we have to check that $\pi^{*}$ is concave and that marginal costs and quantities are positive at the point where the FOC is satisfied. The second order condition is given by

$$
v>\frac{1}{2}\left(\frac{4}{4-\gamma^{2}}\right)^{2},
$$

the condition $x_{S P}^{*}>0$ and $q_{S P}^{*}>0$ yields

$$
v>\frac{4}{(2-\gamma)(2+\gamma)^{2}}
$$


and the condition for $c-x_{S P}^{*}>0$ yields

$$
v>\frac{4 a}{c(2+\gamma)\left(4-\gamma^{2}\right)}
$$

The condition (A.7) implies (A.8) and therefore (A.7) and (A.9) are the binding constraints. Equalizing the terms on the right hand side of these two inequalities yields

$$
\frac{1}{2}\left(\frac{4}{4-\gamma^{2}}\right)^{2}=\frac{4 a}{c(2+\gamma)\left(4-\gamma^{2}\right)}
$$

which can be simplified to

$$
\gamma=\frac{2(a-c)}{a}
$$

One observes the possible relation between $a$ and $c$ for the feasible boundary values of $\gamma$, i.e. $\gamma=0$ and $\gamma=1$. Obviously, $\gamma=0$ implies $a=c$ and $\gamma=1$ implies $a=2 c$. Hence, for $a \geq 2 c$ (A.9) is more restrictive than (A.7) regardless of $\gamma$.One gets immediately

$$
\max \left\{\frac{1}{2}\left(\frac{4}{4-\gamma^{2}}\right)^{2}, \frac{4 a}{c(2+\gamma)\left(4-\gamma^{2}\right)}: \frac{a}{c}>2\right\}=\frac{4 a}{c(2+\gamma)\left(4-\gamma^{2}\right)}
$$

and for $1<\frac{a}{c}<2$ the following holds

$$
\begin{aligned}
& \max \left\{\frac{1}{2}\left(\frac{4}{4-\gamma^{2}}\right)^{2}, \frac{4 a}{c(2+\gamma)\left(4-\gamma^{2}\right)}: 1<\frac{a}{c}<2\right\} \\
&= \begin{cases}\frac{4 a}{c(\gamma+2)\left(4-\gamma^{2}\right)} & \text { for } 0<\gamma \leqslant \frac{2(a-c)}{a} \\
\frac{1}{2}\left(\frac{4}{4-\gamma^{2}}\right)^{2} & \text { for } 1 \geqslant \gamma>\frac{2(a-c)}{a}\end{cases} \\
&<1 .
\end{aligned}
$$

This argumentation directly yields the following result

$$
v> \begin{cases}\frac{4 a}{c(2+\gamma)\left(4-\gamma^{2}\right)} & \text { for } \frac{a}{c} \geqslant 2 \\ 1 & \text { for } 1<\frac{a}{c}<2 .\end{cases}
$$

Finally, it has to be guaranteed that profits of both firms are non-negative for levels of CRA characterized by (A.6). From the expression for $\pi_{S P}^{*}$ given in the Proposition we obtain directly that profits are non-negative iff

$$
v \geq \frac{8}{\left(4-\gamma^{2}\right)^{2}}
$$


and it is easy to see that this inequalitya always holds under condition (??). This establishes that the first order conditions (A.6) indeed characterize the unique subgame perfect equilibrium and the claims of the Proposition now follow by straight-forward calculations.

Q.E.D.

\section{Proof of Proposition 4.2:}

Define

$$
q_{N E}\left(x_{1}, x_{2}\right):= \begin{cases}q_{i}^{*}(\tilde{x}, \tilde{x}) & \text { if } x_{1}=x_{2} \\ \beta & \text { else }\end{cases}
$$

for sufficiently high $\beta$. Because of $\tilde{x} \leq \bar{x}$ we have $\pi_{i}\left(\tilde{x}, \tilde{x}, q_{1}^{*}(\tilde{x}, \tilde{x}), q_{2}^{*}(\tilde{x}, \tilde{x})\right) \geq 0$. Given firm $i$ 's competitor's decision $q_{j}=q_{N E}$ and $x_{j}=\tilde{x}$, we consider two scenarios concerning the strategy of firm $i$ with respect to the $C R A$. If $x_{i} \neq \tilde{x}$ this implies for the reaction of firm $j$ that $\hat{q}_{j}\left(x_{i}, x_{j}\right)=\beta$ which implies $p \leq 0$ and therefore $\pi_{i}<0$. Therefore, in its best response, firm $i$ always chooses $x_{i}=\tilde{x}$. This implies for the best response of firm $i$ with respect to the quantity that

$$
\hat{q}_{i}(\tilde{x}, \tilde{x})=R_{i}^{q}\left(\tilde{x}, q_{j}^{*}(\tilde{x}, \tilde{x})\right)=q_{i}^{*}(\tilde{x}, \tilde{x})
$$

must hold, where $R_{i}^{q}\left(\tilde{x}, q_{j}\right)$ denotes the best reply function of firm $i$ at the quantity choice stage. The crucial equality in (A.10) follows because $q_{i}^{*}(\tilde{x}, \tilde{x})$ is the Nash equilibrium quantity in the second stage for $\left(x_{i}, x_{j}\right)=(\tilde{x}, \tilde{x})$. The quantity choice of firm $i$ at any level of CRA different from $(\tilde{x}, \tilde{x})$ does not affect its payoff and therefore setting these quantities to $\beta$ is (weakly) optimal for the firm. This shows that $\left(x_{N E}, q_{N E}\left(x_{1}, x_{2}\right)\right.$ is indeed a best response for firm $i$.

Q.E.D.

\section{Proof of Proposition 4.3:}

In an interior Nash equilibrium we must have $\hat{q}_{i}=R_{i}^{q}\left(\hat{q}_{j} ; \hat{x}_{1}, \hat{x}_{2}\right)$ and the first order condition with respect to $x_{i}$,

$$
\hat{q}_{i}-v \hat{x}_{i}=0
$$

must hold. It is easy to see that the combination of these two conditions yields under the assumption of positive costs and quantities the unique solution given in (4.5). The second order conditions are $2>0$ and $v>0$, the condition for $\widehat{x}_{N E}^{*}>0, \widehat{q}_{N E}^{*}>0$ is $v>\frac{1}{2+\gamma}$ and the condition for $\widehat{x}_{N E}^{*} \in(0, c)$ can be rewritten to $v>\frac{a}{(2+\gamma) c}$. It is easy to check that under these conditions no equilibrium with zero output of one firm can exist.

Q.E.D. 


\section{Proof of Lemma 4.4:}

We show the Lemma by induction. For $n=0$ we have

$$
\hat{q}^{0}\left(x_{1, t+1}, x_{2, t+1}, q_{1, t}, q_{2, t}, \phi\right)=\phi
$$

which corresponds to (4.6) for $n=0$. Assume that (4.6) holds for some even $n$. Then, we obtain for order $n+1$

$$
\begin{aligned}
\hat{q}^{n+1}\left(x_{1, t+1}, x_{2, t+1}, q_{1, t}, q_{2, t}, \phi\right) \\
=\quad \hat{q}^{n}\left(x_{1, t+1}, x_{2, t+1}, q_{1, t}, q_{2, t}, \alpha q_{j}+(1-\alpha) R_{j}^{q}\left(\phi ; x_{1, t+1}, x_{2, t+1}\right)\right) \\
=\quad\left(\alpha q_{j}+\frac{1}{2}(1-\alpha)\left(a-c+x_{j, t+1}\right)\right) \sum_{k=0}^{\lfloor(n-2) / 2\rfloor}\left(-\frac{\gamma(1-\alpha)}{2}\right)^{2 k} \\
\quad+\left(\alpha q_{i}+\frac{1}{2}(1-\alpha)\left(a-c+x_{i, t+1}\right)\right) \sum_{k=1}^{\lfloor n / 2\rfloor}\left(-\frac{\gamma(1-\alpha)}{2}\right)^{2 k-1} \\
\quad+\left(-\frac{\gamma(1-\alpha)}{2}\right)^{n}\left(\alpha q_{j}+(1-\alpha) \frac{1}{2}\left(a-c+x_{i, t+1}-\gamma \phi\right)\right) \\
=\quad\left(\alpha q_{j}+\frac{1}{2}(1-\alpha)\left(a-c+x_{j, t+1}\right)\right) \sum_{k=0}^{\lfloor n / 2\rfloor}\left(-\frac{\gamma(1-\alpha)}{2}\right)^{2 k} \\
\quad+\left(\alpha q_{i}+\frac{1}{2}(1-\alpha)\left(a-c+x_{i, t+1}\right)\right) \sum_{k=1}^{\lfloor(n+1) / 2\rfloor}\left(-\frac{\gamma(1-\alpha)}{2}\right)^{2 k-1} \\
\quad+\left(-\frac{\gamma(1-\alpha)}{2}\right)^{n+1} \phi .
\end{aligned}
$$

Hence, (4.6) holds also for $n+1$. The transition from an odd $n$ to $n+1$ can be verified analogously, which completes the proof.

Q.E.D.

\section{Proof of Lemma 4.5:}

For given $x_{j, t+1}$ the maximization problem of firm $i$ in stage 1 reads

$$
\begin{gathered}
\max _{x_{i, t+1} \in[0, \bar{X}]}\left[R _ { i } ^ { q } ( q _ { j , t + 1 } ^ { 2 , n e } ; x _ { 1 , t + 1 } , x _ { 2 , t + 1 } ) \left(a-R_{i}^{q}\left(q_{j, t+1}^{2, n e}\left(q_{1, t}, q_{2, t}, x_{1, t+1}, x_{2, t+1}\right) ; x_{1, t+1}, x_{2} t+1\right)\right.\right. \\
\left.\left.-\gamma q_{j, t+1}^{2, n e}-\left(c-x_{i, t+1}\right)\right)-\frac{v}{2} x_{i, t+1}^{2}\right]
\end{gathered}
$$

where it should be kept in mind that $q_{j, t+1}^{2, n e}$ is a function of $\left(q_{1, t}, q_{2, t}, x_{1, t+1}, x_{2, t+1}\right)$. Taking into accout that $R_{i}^{q}$ has to satisfy the first order condition on stage 2 , and assuming that $c-x_{i, t+1}>0$, and $R_{i}^{q}>0$, the first oder condition of this problem 
reads

$$
R_{i}^{q}\left(q_{j, t+1}^{2, n e} ; x_{1, t+1}, x_{2} t+1\right)\left(-\gamma \frac{\partial q_{j, t+1}^{2, n e}}{\partial x_{i, t+1}}+1\right)-v x_{i, t+1}=0
$$

Assuming that the second order condition holds, the first order condition characterizes the optimal solution to this problem as long as the value of $x_{i, t+1}$ satisfying the FOC is positive. From Lemma 4.6 we obtain that

$$
\frac{\partial q_{j, t+1}^{2, n e}}{\partial x_{i, t+1}}=\sum_{k=1}^{\lfloor n / 2\rfloor}\left(-\frac{\gamma(1-\alpha)}{2}\right)^{2 k-1}
$$

and inserting this as well as (4.1) into (A.11) and solving for $x_{i, t+1}$ yields after some tedious calculations the expression given in (4.7). Clearly, this expression is positive for sufficiently large values of $(a-c)$. The second order condition for a maximum is given by

$$
v>\frac{1}{2}\left(1+\sum_{k=1}^{\lfloor n / 2\rfloor}\left(-\frac{\gamma(1-\alpha)}{2}\right)^{2 k}\right)^{2} .
$$

Taking into account that $\left(-\frac{\gamma(1-\alpha)}{2}\right) \in[-0.5,0.5]$ we get for the the right hand side of this inequality

$$
\begin{aligned}
& \frac{1}{2}\left(1+\sum_{k=1}^{\lfloor n / 2\rfloor}\left(-\frac{\gamma(1-\alpha)}{2}\right)^{2 k}\right)^{2} \\
& =\frac{1}{2}\left(1+\left(-\frac{\gamma(1-\alpha)}{2}\right)^{2} \frac{1-\left(-\frac{\gamma(1-\alpha)}{2}\right)^{n}}{1-\left(-\frac{\gamma(1-\alpha)}{2}\right)^{2}}\right)^{2} \\
& <\frac{1}{2}\left(1+\left(\frac{1}{2}\right)^{2} \frac{1+\frac{1}{2}}{1-\left(\frac{1}{2}\right)^{2}}\right)^{2} \\
& =\frac{9}{8}
\end{aligned}
$$

. Since the objective function of firm $i$ on stage 1 is quadratic in $x_{i, t+1}$, the condition $v>\frac{9}{8}$ guarantees the concavity of the objective function on $[0, \bar{X}]$. This completes the proof.

Q.E.D.

\section{Proof of Proposition 4.6:}

It follows directly from the arguments given in the proof of Proposition 3.3 that a symmetric interior fixed point $\left(\hat{x}^{n}, \hat{x}^{n}, \hat{q}_{n}, \hat{q}_{n}\right)$ of the adaptive expectation dynamics 
of order $n$ has to satisfy

$$
\hat{q}^{n}=R^{q}\left(\hat{q}^{n} ; \hat{x}^{n}, \hat{x}^{n}\right)=\frac{a-c+\hat{x}^{n}-\gamma \hat{q}^{n}}{2},
$$

which implies $\hat{q}^{n}=\frac{a-c+\hat{x}^{n}}{2+\gamma}$. Due to the symmetry of the game we have $R_{1}^{x, n}\left(x, \hat{q}^{n}, \hat{q}^{n}, \hat{q}^{n}\right)=$ $R_{2}^{x, n}\left(x, \hat{q}^{n}, \hat{q}^{n}, \hat{q}^{n}\right)$ and we denote this function as $\hat{R}^{x, n}(x)$. Analogously we write $\hat{x}^{1, n e}(x)=x_{i}^{1, n e}\left(x, x, \hat{q}^{n}, \hat{q}^{n}\right), i=1,2$. Since $\hat{R}^{x, n}$ is a contraction also the mapping $\alpha x+(1-\alpha) \hat{R}^{x, n}(x)$ is a contraction for all positive $\alpha$ and, considering the recursion (3.7) used to generate the expectations in stage 1 and the fact that $\hat{R}^{x, n}$ is linear in $x$, we obtain $\left|\frac{\partial \hat{x}^{1, n e}(x)}{\partial x}\right| \leq 1$ for all $x \in(0, \bar{X})$. Furthermore, due to the fixed point property, we must have

$$
\hat{x}^{n}=\hat{R}^{x, n}\left(\hat{x}^{1, n e}\left(\hat{x}^{n}\right)\right)
$$

Taking into account that $\left|\hat{R}^{\prime}\right|<1$ it becomes clear that the function on the the right hand side of this equation is also a contraction, which means that it has a unique fixed point in $[0, \bar{X}]$. Due to the contraction property of $\hat{R}^{x, n}$ also the equation $x=\hat{R}^{x, n}(x)$ has a unique fixed point in $[0, \bar{X}]$. It is easy to see that this fixed point is also a fixed point of (A.13). Therefore, the two fixed points must coincide, which means that $\left(\hat{x}^{n}, \hat{x}^{n}, \hat{q}_{n}, \hat{q}_{n}\right)$ has to satisfy $\hat{x}^{n}=\hat{R}^{x, n}\left(\hat{x}^{n}\right)$ and $\hat{x}^{1, n e}\left(\hat{x}^{n}\right)=\hat{x}^{n}$. Due to the fact that marginal costs of CRAs at $x_{i}=0$ are zero the fixed point can never be at $\hat{x}^{n}=0$. This shows that there exists a unique symmetric interior fixed point and that at this fixed point both firms have correct expectations at both stages.

Concerning items (i) to (iv) in the proposition, the items (i) and (iv) are direct implications of Propositions 3.1 and 3.3 and need no special proof. In order to show points (ii) and (iii) we introduce the following simpliying notation:

$$
F=\frac{-(1-\alpha) \gamma}{2}, \quad S_{1}(n)=\sum_{k=0}^{\lfloor(n-1) / 2\rfloor} F^{2 k}, \quad S_{2}(n)=\sum_{k=1}^{\lfloor n / 2\rfloor} F^{2 k-1}, \quad S_{3}(n)=\sum_{k=0}^{n} F^{k}
$$

From

$$
\hat{x}^{n}=R_{i}^{x, n}\left(\hat{x}^{n}, \hat{q}^{n}, \hat{q}^{n}, \hat{q}^{n}\right)
$$

we obtain after insertion of $\hat{q}^{n}=\frac{a-c+\hat{x}^{n}}{2+\gamma}$ into (4.7) and collecting terms the equation

$$
A(n) \hat{x}^{n}=B(n)(a-c)
$$


with

$$
\begin{aligned}
& A(n)=2 v-1-2 F S_{2}(n)-F^{2} S_{2}(n)^{2} \\
&+\left(1+F S_{2}(n)\right)\left(\frac{\alpha \gamma}{2+\gamma}\left(S_{3}(n)-F^{n}\right)+\frac{\gamma}{2+\gamma} F^{n}-F S_{1}(n)\right) \\
& B(n)=\left(1+F S_{2}(n)\right)\left(S_{3}(n)-\frac{\alpha \gamma}{2+\gamma}\left(S_{3}(n)-F^{n}\right)-\frac{\gamma}{2+\gamma} F^{n}\right) .
\end{aligned}
$$

It can be easily verified that $A(n)$ and $B(n)$ are both positive under our assumptions, which confirms that the equation has a unique positive solution. To prove points (ii) and (iii) of the Proposition we have to characterize the signs of $A(n+1)-A(n)$ and $B(n+1)-B(n)$. To this end we denote $\Delta S_{j}(n)=S_{j}(n+1)-S_{j}(n), j=1, \ldots, 3$ and obtain

$$
\begin{aligned}
\Delta S_{1}(n) & = \begin{cases}F^{n} & n \text { even } \\
0 & n \text { odd }\end{cases} \\
\Delta S_{2}(n) & = \begin{cases}0 & n \text { even } \\
F^{n} & n \text { odd }\end{cases} \\
\Delta S_{3}(n) & =F^{n+1}
\end{aligned}
$$

Considering first the case where $n$ is even we have after collecting terms

$$
\begin{aligned}
& A(n+1)-A(n)=\left(1+F S_{2}(n)\right) F^{n}\left(\frac{\gamma}{2+\gamma}(F-1)-F+\frac{\alpha \gamma}{2+\gamma}\right)=0 \\
& B(n+1)-B(n)=\left(1+F S_{2}(n)\right) F^{n}\left(F-\frac{\alpha \gamma}{2+\gamma}-\frac{\gamma}{2+\gamma}(F-1)\right)=0,
\end{aligned}
$$

where $\left(F-\frac{\alpha \gamma}{2+\gamma}-\frac{\gamma}{2+\gamma}(F-1)\right)=0$ can be verified by inserting $F=\frac{-(1-\alpha) \gamma}{2}$. 
Clearly, this implies that $\hat{x}^{n+1}=\hat{x}^{n}$. If $n$ is odd the corresponding terms read

$$
\begin{aligned}
& A(n+1)-A(n)=\left(1+F S_{2}(n)\right) F^{n} \underbrace{\left(-2 F+\frac{\alpha \gamma}{2+\gamma}+\frac{\gamma}{2+\gamma}(F-1)\right)}_{=-F} \\
& +F^{n+1}\left(-F^{n+1}+\frac{\alpha \gamma}{2+\gamma}\left(S_{3}(n)-F^{n}\right)+\frac{\gamma}{2+\gamma} F^{n}-F S_{1}(n)\right) \\
& =F^{n+1}(-1-F \underbrace{\left(S_{1}(n)+S_{2}(n)\right)}_{=S_{3}(n)-F^{n}}+\frac{\alpha \gamma}{2+\gamma} S_{3}(n)+\frac{(1-\alpha) \gamma}{2+\gamma} F^{n}-F^{n+1}) \\
& =\underbrace{F^{n+1}}_{>0}(\underbrace{-1+S_{3}(n)\left(\frac{\alpha \gamma}{2+\gamma}-F\right)}_{<0}+\frac{(1-\alpha) \gamma}{2+\gamma} \underbrace{F^{n}}_{<0}) \\
& <0
\end{aligned}
$$

and

$$
\begin{aligned}
B(n+1)-B(n)= & \left(1+F S_{2}(n)\right) F^{n} \underbrace{\left(F-\frac{\alpha \gamma}{2+\gamma}-\frac{\gamma}{2+\gamma}(F-1)\right)}_{=0} \\
& +F^{n+1}\left(S_{3}(n)-\frac{\alpha \gamma}{2+\gamma}\left(S_{3}(n)-F^{n}\right)-\frac{\gamma}{2+\gamma} F^{n}\right) \\
= & \underbrace{F^{n+1}}_{>0}(\underbrace{S_{3}(n)\left(1-\frac{\alpha \gamma}{2+\gamma}\right)}_{>0} \underbrace{-\frac{(1-\alpha) \gamma}{2+\gamma} F^{n}}_{>0}) \\
>0 . & .
\end{aligned}
$$

This implies that $\hat{x}^{n+1}=\frac{B(n+1)}{A(n+1)}>\frac{B(n)}{A(n)}=\hat{x}^{n}$ and therefore also $\hat{q}^{n+1}>\hat{q}^{n}$ must hold.

Q.E.D. 